\title{
DNGR1-mediated deletion of A20/Tnfaip3 in dendritic cells alters T and B- cell homeostasis and promotes autoimmune liver pathology
}

\author{
Tridib Das ${ }^{\mathrm{a}}$, Ingrid M. Bergen ${ }^{\mathrm{a}}$, Thomas Koudstaal ${ }^{\mathrm{a}}$, Jennifer A.C. van Hulst ${ }^{\mathrm{a}}$, Geert van Loo ${ }^{\mathrm{b}, \mathrm{c}}$, \\ André Boonstra $^{\mathrm{d}}$, Thomas Vanwolleghem ${ }^{\mathrm{d}, \mathrm{e}}$, Patrick S.C. Leung ${ }^{\mathrm{f}}$, M. Eric Gershwin ${ }^{\mathrm{f}}$, \\ Rudi W. Hendriks ${ }^{a}$, Mirjam Kool ${ }^{a, *}$

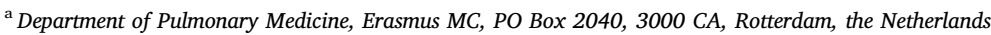 \\ ${ }^{\mathrm{b}}$ VIB Center for Inflammation Research, VIB, Technologiepark 71, 9052, Ghent, Belgium \\ ${ }^{\mathrm{c}}$ Department of Biomedical Molecular Biology, Ghent University, Technologiepark 71, 9052, Ghent, Belgium \\ ${ }^{\mathrm{d}}$ Department of Gastroenterology and Hepatology, Erasmus MC, PO Box 2040, 3000 CA, Rotterdam, the Netherlands \\ ${ }^{\mathrm{e}}$ Department of Gastroenterology and Hepatology, University Hospital Antwerp, Wilrijkstraat 10, 2650, Edegem, Belgium \\ ${ }^{\mathrm{f}}$ Division of Rheumatology/Allergy and Clinical Immunology, University of California, 451 Health Sciences Drive, Suite 6510, Davis, CA 95616, USA
}

\section{A B S T R A C T}

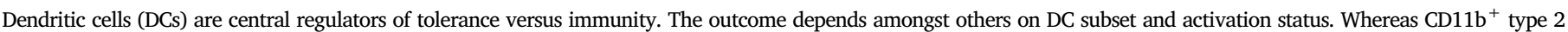

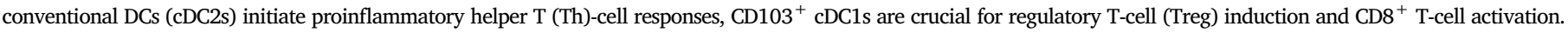

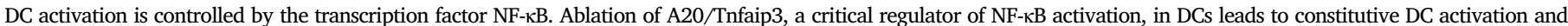
development of systemic autoimmunity. We hypothesized that the activation status of cDCs controls the development of autoimmunity.

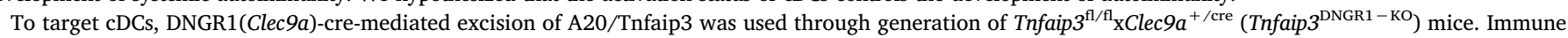
cell activation was evaluated at 31-weeks of age.

We found that DNGR1-cre-mediated deletion of A20/Tnfaip3 resulted in liver pathology characterized by inflammatory infiltrates adjacent to the portal triads.

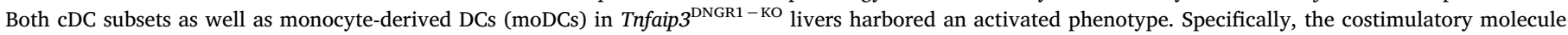

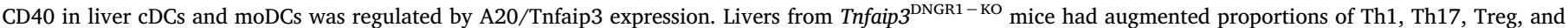

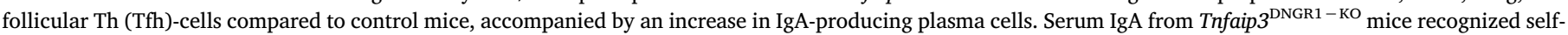
proteins, specifically cytoplasmic proteins in liver periportal regions.

These data show that enhanced activation of cDCs and moDCs, due to A20/Tnfaip3 ablation, promotes the development of organ-specific autoimmunity but not systemic autoimmunity. This model could be useful to examine the pathobiological processes contributing to autoimmune liver diseases.

\section{Introduction}

The adaptive immune response is critically altered in autoimmune diseases, whereby activation of pathogenic T-cells is induced by dendritic cells (DCs). DCs are known to serve as central regulators in the delicate balance between tolerance and immunity [1]. During steady state, immature DCs present self-antigens to T-cells, thereby inducing regulatory $\mathrm{CD}^{+}{ }^{+} \mathrm{T}$-cell (Tregs), T-cell anergy or autoreactive T-cell deletion [2-4]. These mechanisms prevent T-cell mediated autoimmunity [5]. The balance between tolerance and immunity depends on the maturation status of DCs, a process that is strictly regulated [6,7]. During an immune response, DCs are activated through ligand-receptor interactions that induce activation of the NF- $\kappa \mathrm{B}$ pathway and provoke proinflammatory cytokine production [8]. NF- $\mathrm{\kappa B}$ activation is tightly controlled by several mechanisms. One major inhibitor of NF- $\mathrm{KB}$ signalling is the ubiquitin-editing enzyme TNFa-induced protein 3 (TNFAIP3) or A20 [9]. DC-specific deletion of Tnfaip3/A20 using the Cd11c promotor in mice (Tnfaip $3^{\mathrm{CD} 11 \mathrm{c}-\mathrm{KO}}$ mice) resulted in spontaneous activation of DCs and induction of autoreactive $\mathrm{CD}^{+}{ }^{+} \mathrm{T}$ helper (Th)1-cells and Th17cells differentiation, causing a severe and complex autoimmune inflammatory phenotype $[10,11]$. Tnfaip $3^{\mathrm{CD} 11 \mathrm{c}-\mathrm{KO}}$ mice developed features of inflammatory bowel disease (IBD) [10] and systemic lupus erythematosus (SLE) [11]. Importantly, genetic polymorphisms in the TNFAIP3 gene are associated with several human autoimmune disorders [12,13].

DCs comprise different subsets with specialized functions [5]. Both

\footnotetext{
* Corresponding author.

E-mail addresses: t.das@erasmusmc.nl (T. Das), i.bergen@erasmusmc.nl (I.M. Bergen), t.koudstaal.1@erasmusm.nl (T. Koudstaal), j.vanhulst@erasmusmc.nl (J.A.C. van Hulst), geert.vanloo@irc.vib-ugent.be (G. van Loo), p.a.boonstra@erasmusmc.nl (A. Boonstra), t.vanwolleghem@erasmusmc.nl (T. Vanwolleghem), psleung@ucdavis.edu (P.S.C. Leung), megershwin@ucdavis.edu (M.E. Gershwin), r.hendriks@erasmusmc.nl (R.W. Hendriks),m.kool@erasmusmc.nl (M. Kool).
} 
conventional DCs (cDCs) and plasmacytoid DCs (pDCs) are present during steady state. $\mathrm{CD} 103^{+} / \mathrm{CD}^{+}$type $1 \mathrm{cDCs}(\mathrm{cDC} 1 \mathrm{~s})$ are important for peripheral tolerance as they can present tissue-associated self-antigens [5]. During steady state, cDC1s can induce Tregs [14-16], Th-cell deletion [17], $\mathrm{CD}^{+} \mathrm{T}$-cell tolerance $[17,18]$, and once activated, cDC1s also provoke cytotoxic T-cell responses [19]. Strikingly, ablation of cDC1s does not cause spontaneous autoimmunity [19,20], it only alters intestinal T-cell homeostasis [21]. Under steady state, CD11b ${ }^{+}$/ $\mathrm{CD}^{+}{ }^{+}$type 2 cDCs (cDC2s) also induce T-cell tolerance [22], and provoke Treg proliferation and differentiation [16,23]. Activated cDC2s strongly promote Th-cell activation and induce Th-cell differentiation into Th2 [24] or Th17-cells [25]. During inflammation, monocyte-derived DCs (moDCs) arise and produce chemokines to attract immune cells to the inflammatory lesion [26].

It is unclear what the contribution of different DC subsets is to the autoimmune phenotype in Tnfaip $3^{\mathrm{CD} 11 \mathrm{c}-\mathrm{KO}}$ mice. As $\mathrm{cDCs}$ are important instructors of T-cell tolerance, we hypothesized that cDCs crucially contribute to the autoimmune phenotype. To determine cDC function, we used DNGR1-cre-driven [27] deletion of Tnfaip3/A20 (Tnfaip $3^{\mathrm{DNGR} 1-\mathrm{KO}}$ mice) as DNGR1-cre can induce efficient deletion of target genes in $\sim 95 \%$ of organ cDC1s, $25-40 \%$ of cDC2s, and $\sim 5-25 \%$ of moDCs [27].

\section{Material \& methods}

\subsection{Mice}

Male and female mice harbouring a conditional Tnfaip3 allele flanked by LoxP sites [28] were crossed to mice expressing the Cre recombinase under the Clec9a promotor (DNGR1) [27], generating Tnfaip $3^{\mathrm{fl} / \mathrm{fl}} \times$ Clec $9 a^{+/ \mathrm{cre}}$ mice (Tnfaip $3^{\mathrm{DNGR} 1-\mathrm{KO}}$ mice). Mice were $>10$ times backcrossed to obtain a C57Bl/6 background. Tnfaip $3^{\text {fl/ }}$ ${ }^{\mathrm{fl}} \mathrm{xClec} 9 a^{+/+}$littermates (Tnfaip3 ${ }^{\mathrm{DNGR} 1-\mathrm{WT}}$ mice) served as controls. All mice were sacrificed between 11 and 31 weeks of age.

To trace Tnfaip3-deletion and function in cDCs, we crossed

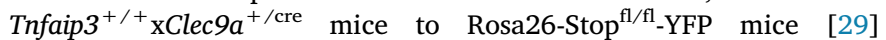
(Tnfaip $3^{\text {DNGR1-ROSA-WT }}$ mice) and Tnfaip $3^{\mathrm{fl} / \mathrm{fl}} \mathrm{xClec} 9 a^{+/ \text {cre }}$ mice to Rosa26-Stop ${ }^{\mathrm{fl} / \mathrm{fl}}$-YFP mice (Tnfaip $3^{\text {DNGR1-ROSA-KO }}$ mice). Mice were housed under specific pathogen-free conditions and had ad libitum access to food and water. All experiments were approved by the animal ethical committee of the Erasmus MC, Rotterdam, The Netherlands and comply to the EU Directive 2010/63/EU for animal experiments.

\subsection{Cell suspension preparation}

Spleen and liver were isolated and used for flow cytometry. Spleens were homogenized through a $100-\mu \mathrm{m}$ cell strainer. Erythroid cells present in the spleen cell suspensions were lysed using osmotic lysis buffer $\left(8.3 \% \mathrm{NH}_{4} \mathrm{CL}, 1 \% \mathrm{KHCO}_{3}\right.$, and $0.04 \% \mathrm{NA}_{2}$ EDTA in Milli-Q). Liver single-cell suspensions were obtained, as previously described [30], by digesting with Liberase TM (Roche, Basel, Switzerland) for $30 \mathrm{~min}$ at $37^{\circ} \mathrm{C}$. After digestion, the livers were homogenized using a $100-\mu \mathrm{m}$ cell strainer (Fischer Scientific). Hepatocytes were discarded using two low speed centrifuge steps. Lastly, erythroid cells were lysed using osmotic lysis buffer.

\subsection{Flow cytometry procedures}

Flow cytometry surface and intracellular staining procedures have been described previously [31]. Monoclonal antibodies used for flow cytometric analyses are listed in Supplementary Table 1. For all experiments, dead cells were excluded using fixable Amcyan viability dye (eBioscience, San Diego, CA, USA). To measure cytokine production, cells were stimulated with $10 \mathrm{ng} / \mathrm{mL}$ PMA (Sigma-Aldrich, St. Louis, MI, USA) and $250 \mathrm{ng} / \mathrm{mL}$ ionomycin (Sigma-Aldrich) in the presence of GolgiStop (BD Biosciences, San Jose, CA, USA) for $4 \mathrm{~h}$ at $37^{\circ} \mathrm{C}$. Flow cytometry absolute counting beads (Polysciences, Warrington, PA, USA) were added to liver flow cytometry samples. Data were acquired using an LSR II flow cytometer (BD Biosciences) with FACS Diva ${ }^{\mathrm{TM}}$ software and analyzed by FlowJo version 9 (Tree Star Inc software, Ashland, OR, USA).

\subsection{Liver histology}

The right lobe of the liver was fixated with 4\% PFA (Carl Roth, Karlsruhe, Germany) for $24 \mathrm{~h}$ before paraffin embedding. Six- $\mu \mathrm{m}$-thick paraffin embedded liver sections were stained with hematoxylin and eosin, and using Sirius Red (Sigma-Aldrich) and Fast Green (SigmaAldrich) to stain for collagen fibers, as previously described [32]. Liver pathology was scored using the histopathologic scoring system according to Ishak et al. [33] For immunohistochemical stainings, antigen retrieval on paraffin-sections was established using citrate buffer (Sigma-Aldrich).

Paraffin sections were stained for Cytokeratin 7, CD3 and B220. The primary antibodies used for immunohistochemistry are listed in Supplementary Table 2. Sections were incubated for $1 \mathrm{~h}$ with the primary antibodies. After washing, slides were incubated for $30 \mathrm{~min}$ with secondary antibodies (Supplementary Table 2). On paraffin sections which were stained for Cytokeratin 7 and CD3, the anti-Rabbit ABC Peroxidase Kit was utilized (Vector Labs, Burlingame, CA, USA). Diaminobenzene (DAB) and Fast Blue Alkaline phosphatase substrates were used to retrieve specific staining.

\subsection{Serum measurements}

To determine liver function, aspartate aminotransferase (AST) and alanine aminotransferase (ALT) enzymes were measured in serum.

For total immunoglobulin concentrations, Nunc Microwell plates (Life technologies, Carlsbad, CA, USA) were coated with $1 \mu \mathrm{g} / \mathrm{ml}$ goatanti-mouse IgM, IgA, IgG1, IgG2a, IgG2b, or IgG3 (Southern Biotech, Birmingham, AL, USA) overnight at $4 \mathrm{C}^{\circ}$. Wells were blocked with $10 \%$ FCS (Capricorn Scientific, Ebsdorfergrund, Germany) in PBS (Thermo Scientific, Waltham, MA, USA) for $1 \mathrm{~h}$. Standards and serum were diluted in PBS and incubated for $3 \mathrm{~h}$ at room temperature. Depending on the isotype of interest, anti-mouse biotin-labeled IgM, IgA, IgG1, IgG2a, IgG2b, or IgG3 (Southern Biotech) was incubated for $1 \mathrm{~h}$. StreptavidinHRP (eBioscience) and TMB substrate (eBioscience) was used to develop the ELISA and then optical density (OD) was measured at $450 \mathrm{~nm}$ on a Microplate Reader (Bio-Rad, Hercules, CA, USA).

For detection of anti-cardiolipin antibodies, Nunc Microwell plates were coated with $10 \mu \mathrm{g} / \mathrm{ml}$ cardiolipin from bovine heart (Sigma) in ethanol and left to dry overnight. For detection of dsDNA, $20 \mu \mathrm{g} / \mathrm{ml}$ dsDNA from calf thymus (Sigma) was coated overnight on pre-coated poly-1-lysine microwells. Wells were blocked with $2 \%$ BSA/PBS for $2 \mathrm{~h}$, after which serum was incubated for $2 \mathrm{~h}$. Anti-mouse IgG1 biotin/ streptavidin-HRP (eBioscience) was used to develop the ELISA with TMB substrate (eBioscience). Detection of immunoglobulin IgG/IgA versus PDC-E2, sp100 and gp210 was performed as previously described [34].

For detection of autoreactive IgA binding to tissues, we used $5 \mu \mathrm{m}$ cryo-sectioned liver and pancreas from $\operatorname{Rag} 1^{\mathrm{KO}}$ mouse [35], as these mice lack mature B-cells and consequently endogenous immunoglobulins are absent. After $10 \mathrm{~min}$ acetone fixation (Sigma) and 10 min block with $10 \%$ normal goat serum (NGS), sera from Tnfaip $3^{\text {DNGR1-WT }}$ mice (dilution $1 / 33$ ) and Tnfaip $3^{\text {DNGR1-KO }}$ mice (dilution $1 / 100$ ) were incubated for $1 \mathrm{~h}$. Different dilutions were used to correct for total IgA concentrations in serum from Tnfaip $3^{\text {DNGR1 - WT }}$ and Tnfaip $3^{\text {DNGR1-KO }}$ mice. Incubation with anti-mouse IgA biotin/streptavidin (BD) and subsequently goat anti-Rat-AP (Sigma), followed by New Fuchsine (Sigma) staining were used to visualize liver-specific IgA. Slides were counterstained with Gills hematoxylin (Sigma). 


\subsection{Statistics}

Statistical significance of data was calculated using the non-parametric Mann Whitney U test. P-values $<0.05$ were considered significant. All analyses were performed using Prism (GraphPad Software version 9, La Jolla, CA, USA). All data are presented as the mean with the standard error of the mean (SEM).

\section{Results}

\subsection{Tnfaip $3^{D N G R 1-K O}$ mice have spontaneous periportal liver infiltrates} and signs of chronic inflammation

To investigate whether immune homeostasis is altered when cDCs harbor a DNGR1-mediated deletion of the A20/Tnfaip3 gene, we evaluated 31-week-old Tnfaip3 $3^{\text {DNGR1 }}$ mice. Tnfaip $3^{\text {DNGR1-KO }}$ mice had splenomegaly and hyper cellularity in contrast to Tnfaip $3^{\text {DNGR1-WT }}$ littermate controls (Supplementary Fig. 1A). Splenic DC and T-cell numbers did not differ between Tnfaip $3^{\text {DNGR1 - KO }}$ mice and Tnfaip $3^{\text {DNGR1-WT }}$ mice (Supplementary Figs. 1B-C). Mainly marginal zone B-cells contributed to the increase of total splenic B-cells numbers in Tnfaip3 $3^{\text {DNGR1 - KO }}$ mice compared to Tnfaip $3^{\text {DNGR1-WT }}$ mice (Supplementary Fig. 1D).

We next evaluated kidneys, pancreas, intestines, and livers in 31week-old Tnfaip $3^{\text {DNGR1 - KO }}$ mice for signs of inflammation. Spleens of Tnfaip $3^{\text {DNGR1 - KO }}$ mice showed mild architectural changes of the white pulp lymphoid follicles in comparison to WT mice (Supplementary Fig. 2A). Pancreas, terminal ileum, colon, and kidneys of Tnfaip $3^{\text {DNGR1 - KO }}$ mice did not show any sign of inflammation or remodelling (Supplementary Figs. 2B-E). In contrast, livers of all Tnfaip $3^{\text {DNGR1-KO }}$ mice showed periportal inflammatory infiltrates at 31-weeks of age compared to WT mice (Fig. 1A/B). Mild interface hepatitis (also known as piecemeal necrosis) and focal necrosis with inflammation, which are often seen in autoimmune hepatitis (AIH) [36], were observed in Tnfaip $3^{\text {DNGR1-KO }}$ mice, but not in WT mice (Fig. 1C/ D). Mild liver fibrosis occurred around portal triads of Tnfaip $3^{\text {DNGR1-KO }}$ mice compared to controls (Fig. 1E/F). These features resulted in a significantly higher liver histopathologic score for Tnfaip $3^{\text {DNGR1-KO }}$ mice compared to WT mice (Fig. 1G). Also increased cytokeratin 7 expression, a protein expressed in bile ducts and indicative for ductular reaction, could be observed in Tnfaip $3^{\mathrm{DNGR} 1-\mathrm{KO}}$ mice compared to littermate controls (Fig. 1H). Furthermore, a mild, but significant, increase in serum aspartate aminotransferase (AST) was observed in Tnfaip $3^{\text {DNGR1-KO }}$ mice compared to WT controls (Fig. 1I). No differences were observed between male or female Tnfaip $3^{\text {DNGR1-KO }}$ mice (data not shown).

Summarizing, these data illustrate that aged Tnfaip $3^{\mathrm{DNGR} 1-\mathrm{KO}}$ mice develop a spontaneous liver pathology characterized by the presence of periportal inflammatory infiltrates and signs of chronic inflammation.

\subsection{DNGR1-mediated deletion of A20/Tnfaip3 targets both $c D C s$ and moDCs}

In line with histological findings, the total number of liver CD45 ${ }^{+}$

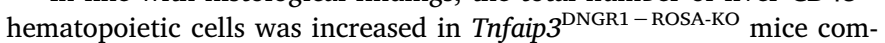
pared to Tnfaip $3^{\text {DNGR1-ROSA-WT }}$ mice (Fig. 2A). Within liver CD45 ${ }^{+}$ cells, we determined the different DC subsets (Fig. 2B). The proportion of DCs from total CD $45^{+}$cells was lower in Tnfaip $3^{\text {DNGR1-ROSA-KO }}$ mice compared to Tnfaip $3^{\text {DNGR1-ROSA-WT }}$ mice, but total liver DC numbers were not significantly different (Fig. 2C). Conventional DC1s and cDC2s were neither significantly altered as proportions of DCs nor in cell numbers between the two genotypes (Fig. 2D/E). To investigate DNGR1-cre mediated deletion efficiency and the effect of A20/Tnfaip3 ablation on liver cDC homeostasis, we examined Tnfaip $3^{\text {DNGR1 - ROSA-WT }}$ mice and Tnfaip $3^{\text {DNGR1-ROSA-KO }}$ mice, in which YFP expression can be used as a lineage tracer of DNGR1 expression. Similar to other organs [27], in livers of Tnfaip $3^{\text {DNGR1-ROSA-WT }}$ mice $~ 95 \%$ of cDC1s showed YFP expression, which was reduced to $\sim 55 \%$ in Tnfaip $3^{\text {DNGR1-ROSA-KO }}$ mice (Fig. 2F). YFP expression in liver cDC2s did not differ between Tnfaip $3^{\text {DNGR1-ROSA-WT }}$ mice and Tnfaip $3^{\text {DNGR1-ROSA-KO }}$ mice and remained $\sim 35 \%$ (Fig. $2 \mathrm{~F}$ ). The absolute number of $\mathrm{YFP}^{+} \mathrm{cDC1}^{\mathrm{s}}$ was similar between Tnfaip $3^{\text {DNGR1-ROSA-WT }}$ mice and Tnfaip $3^{\text {DNGR1-ROSA-KO }}$ mice (Fig. 2G). Plasmacytoid DCs in liver were unaffected by DNGR1mediated deletion $\left(\sim 2 \% \mathrm{YFP}^{+}\right.$; data not shown). We next determined Kupffer cells and monocyte-derived DCs (moDCs) (Fig. 2H). The total number of both Kupffer cells and moDCs was increased in Tnfaip $3^{\text {DNGR1 - KO }}$ mice compared to Tnfaip $3^{\text {DNGR1-WT }}$ mice (Fig. 2I). While Kupffer cell proportions of $\mathrm{CD}_{4} 5^{+}$cells were similar, moDCs significantly expanded as a proportion of $\mathrm{CD}^{2} 5^{+}$cells in Tnfaip $3^{\text {DNGR1-KO }}$ mice compared to littermate controls (Fig. 2J). YFP expression was almost absent in Kupffer cells $(<1 \%)$ in both genotypes (Fig. 2K). Liver moDCs, albeit present in low numbers in Tnfaip $3^{\text {DNGR1-ROSA-WT }}$ mice, harbored a slightly higher YFP expression WT mice $(\sim 45 \%)$ compared to and in Tnfaip $3^{\text {DNGR1-ROSA-WT }}$ and Tnfaip $3^{\text {DNGR1-ROSA-KO }}$ mice ( $\sim 30 \%$ ) (Fig. $2 \mathrm{~K}$ ).

Concluding, DNGR1-lineage tracing indicated that in livers of control mice respectively $95 \%$ and $35 \%$ of $\mathrm{cDC} 1 \mathrm{~s}$ and cDC2s, as well as $45 \%$ of moDCs express or once expressed DNGR1. Furthermore, due to deletion of A20/Tnfaip3 55\% and 35\% of liver cDC1s and cDC2s, along with $30 \%$ of liver moDCs were affected by DNGR1-targeting.

\subsection{Surface CD4O expression is increased through both cell-intrinsic and cell-extrinsic effects of Tnfaip3-deficiency in cDC1s, cDC2s, and moDCs}

In livers of Tnfaip $3^{\mathrm{DNGR} 1-\mathrm{KO}}$ mice, both $\mathrm{cDC} 1 \mathrm{~s}$ and $\mathrm{cDC} 2 \mathrm{~s}$ showed significantly enhanced surface expression of the costimulatory molecule CD40 compared to Tnfaip $3^{\mathrm{DNGR} 1-\mathrm{WT}}$ mice (Fig. 3A/B). In Tnfaip $3^{\text {DNGR1-WT }}$ mice, expression of the co-inhibitory molecule PD-L1 was higher in cDC2s than in cDC1s (Fig. 3B). Both cDC1s and cDC2s in Tnfaip $3^{\text {DNGR1-KO }}$ mice significantly increased PD-L1 expression in comparison to Tnfaip $3^{\text {DNGR1-WT }}$ mice (Fig. 3A/B). No differences were observed for MHC-I or MHC-II expression in cDCs (data not shown).

We next analyzed whether the altered co-stimulatory molecule expression was a direct consequence of A20/Tnfaip3-deletion, and compared $\mathrm{YFP}^{+}$and $\mathrm{YFP}^{-}$cDCs, indicative of A20/Tnfaip3-deficient or A20/Tnfaip3-sufficient cDCs respectively in Tnfaip3 $3^{\text {DNGR1-ROSA-KO }}$ mice. $\mathrm{YFP}^{+} \mathrm{cDC}^{\mathrm{s}}$ and $\mathrm{YFP}^{+}$cDC2s from Tnfaip $3^{\text {DNGR1-ROSA-KO }}$ mice harboured significantly higher $\mathrm{CD} 40$ expression in comparison to $\mathrm{YFP}^{-}$ cDCs within the same livers (Fig. 3C), whereas PD-L1 expression was similar in $\mathrm{YFP}^{+}$and $\mathrm{YFP}^{-}$cDC1s/cDC2s from Tnfaip $3^{\text {DNGR1-ROSA-KO }}$ mice (Fig. 3D). CD40 and PD-L1 expression was already enhanced on YFP-negative Tnfaip3-sufficient cDC1s/cDC2s from Tnfaip $3^{\text {DNGR1-ROSA- }}$ ко mice compared to Tnfaip3-sufficient cDC1s/cDC2s in Tnfaip $3^{\text {DNGR1-ROSA-WT }}$ mice (Fig. 3C/D). CD40 expression on liver moDCs did not differ between Tnfaip $3^{\text {DNGR1 - KO }}$ mice and WT controls (Fig. 3E/F). In contrast, $\mathrm{YFP}^{+}$moDCs of Tnfaip $3^{\text {DNGR1-ROSA-KO mice }}$ showed a higher CD40 expression than $\mathrm{YFP}^{-}$moDCs (Fig. 3G/H). Liver moDCs also harboured higher PD-L1 expression in Tnfaip $3^{\text {DNGR1-KO }}$ mice compared to Tnfaip $3^{\text {DNGR1-WT }}$ controls (Fig. 3E/F), but this did not differ between $\mathrm{YFP}^{+}$or $\mathrm{YFP}^{-}$moDCs in Tnfaip $3^{\mathrm{DNGR} 1-\mathrm{ROSA}-\mathrm{KO}}$ mice (Fig. 3G/H).

In summary, both liver cDC1s and cDC2s of Tnfaip $3^{\mathrm{DNGR} 1-\mathrm{KO}}$ mice show an activated phenotype, e.g. increased CD40 and PD-L1 expression, irrespective of Tnfaip3 deletion. Only CD40 expression is specifically enhanced due to cell-intrinsic loss of Tnfaip3. Liver moDCs of Tnfaip $3^{\text {DNGR1-KO }}$ mice show elevated PD-L1 expression compared to 
A

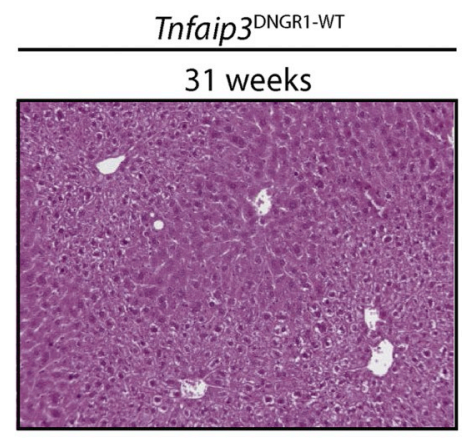

C

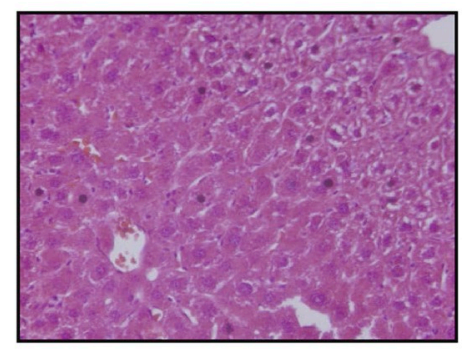

E
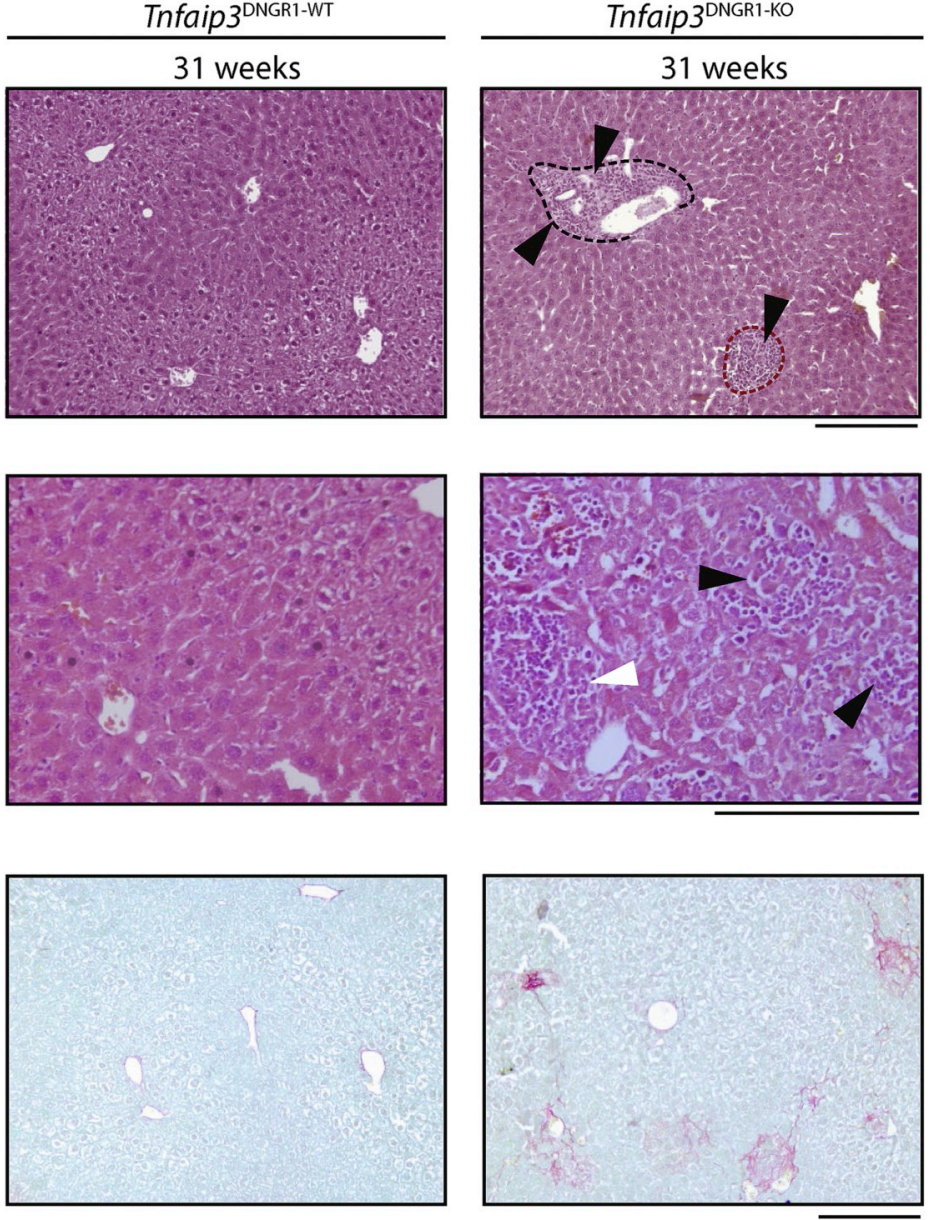

D

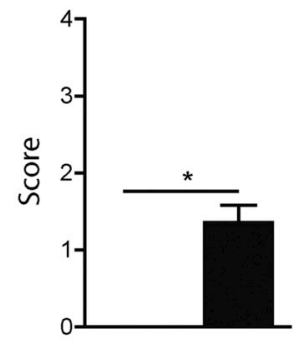

$\mathbf{F}$

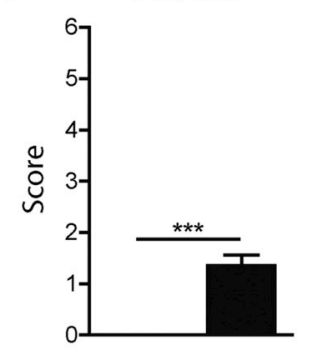

I
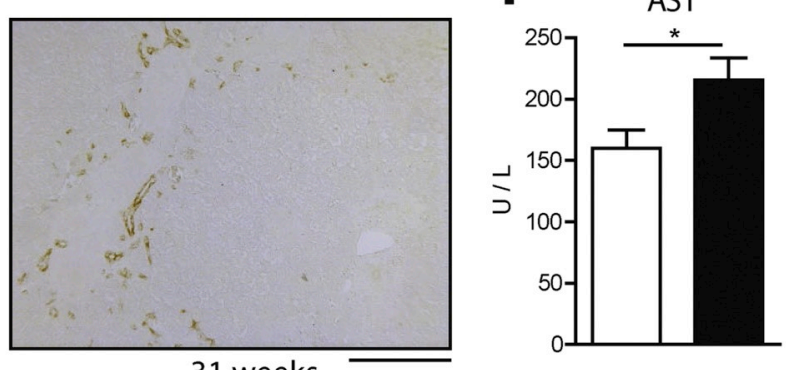

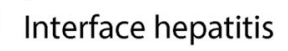

Fibrosis

Portal inflammation

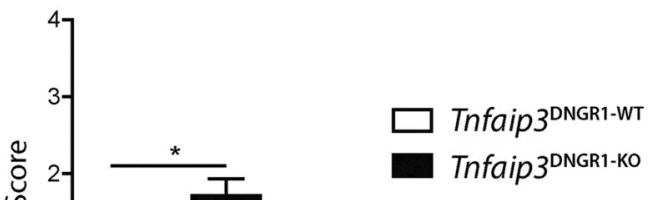

Focal necrosis

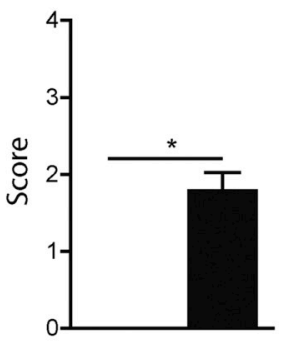

G

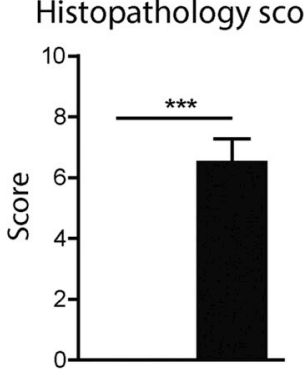

ALT

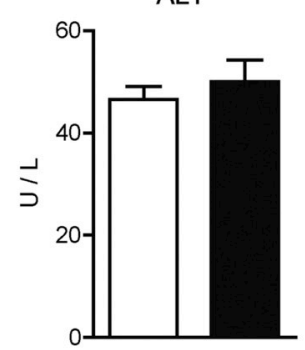

31 weeks

Fig. 1. Tnfaip $3^{\mathrm{DNGR} 1-\text { КО }}$ mice have spontaneous periportal liver infiltrates and signs of chronic inflammation. Tnfaip $3^{\mathrm{DNGR} 1-\mathrm{WT}}$ mice and Tnfaip $3^{\mathrm{DNGR} 1-\mathrm{KO}}$ mice were analyzed at 31-weeks of age. (A) Hematoxylin and eosin (H\&E) stained liver histology with periportal infiltrates (black dashed line) and lobular infiltrates (red dashed line). (B) Quantification of the portal inflammation score (max. 4). (C) Larger magnification H\&E stained liver histology indicating areas of interface hepatitis (white arrow) and focal necrosis with inflammation (black arrows). (D) Quantified interface hepatitis and focal necrosis with inflammation score (max. 4). (E-F) Sirius Red staining to stain collagen fibers (E) and enumeration of the resulting fibrosis score (max. 6) (F). (G) Quantification of the total histopathology score (max. 18). (H) Immunohistochemistry of livers for Cytokeratin 7 (brown). (I) Quantification of serum liver enzymes AST and ALT in 31-week-old Tnfaip $3^{\text {DNGR1-WT }}$ mice and Tnfaip $3^{\mathrm{DNGR} 1-\mathrm{KO}}$ mice. Scale bars represent $200 \mu \mathrm{m}$. Results of pooled data from 3 experiments and are presented as mean \pm SEM of $\mathrm{n}=15$ mice per group. $* \mathrm{P}<0.05,{ }^{* * *} \mathrm{P}<0.001$. (For interpretation of the references to colour in this figure legend, the reader is referred to the Web version of this article.)

Tnfaip3 $3^{\text {DNGR1 - WT }}$ mice. Strikingly, enhanced CD40 expression is specifically observed in Tnfaip3-deficient moDCs.

3.4. Livers of Tnfaip $3^{\text {DNGR1-KO }}$ mice have increased proportions of Th1cells, Th17-cells and Tregs

The proportion of $\mathrm{CD}^{+}{ }^{+}$T-cells and natural killer (NK)-cells within $\mathrm{CD} 45^{+}$cells were elevated in the livers of Tnfaip $3^{\mathrm{DNGR} 1-\mathrm{KO}}$ mice compared to control mice (Fig. 4A). The percentages of $\mathrm{CD}^{+}{ }^{+}$Th-cells and neutrophils were reduced in Tnfaip $3^{\mathrm{DNGR} 1-\mathrm{KO}}$ mice compared to Tnfaip $3^{\text {DNGR1-WT }}$ controls (Fig. 4A). Liver F4/80 ${ }^{+}$macrophages and Kupffer cells, monocytes, and B-cell percentages were not significantly different (Fig. 4A). Due to an increase in absolute numbers of $\mathrm{CD}^{4} 5^{+}$ hematopoietic cells (Fig. 2A), the total number of CD8 ${ }^{+}$T-cells, CD4 ${ }^{+}$ Th-cells, and B-cells were significantly increased in Tnfaip $3^{\mathrm{DNGR} 1-\mathrm{KO}}$ mice compared to controls (Fig. 4B). Clusters of T-cells and B-cells were

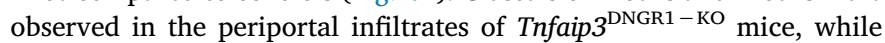
only occasionally among hepatocytes in Tnfaip $3^{\mathrm{DNGR} 1-\mathrm{WT}}$ mice 
A

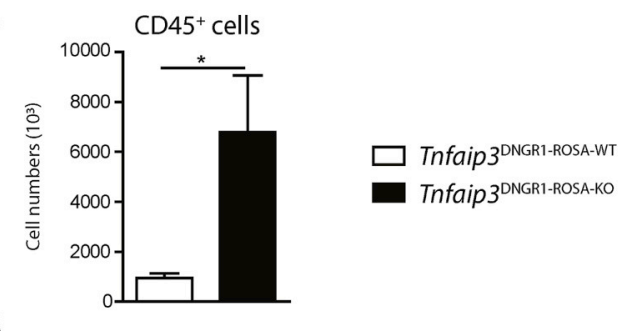

B

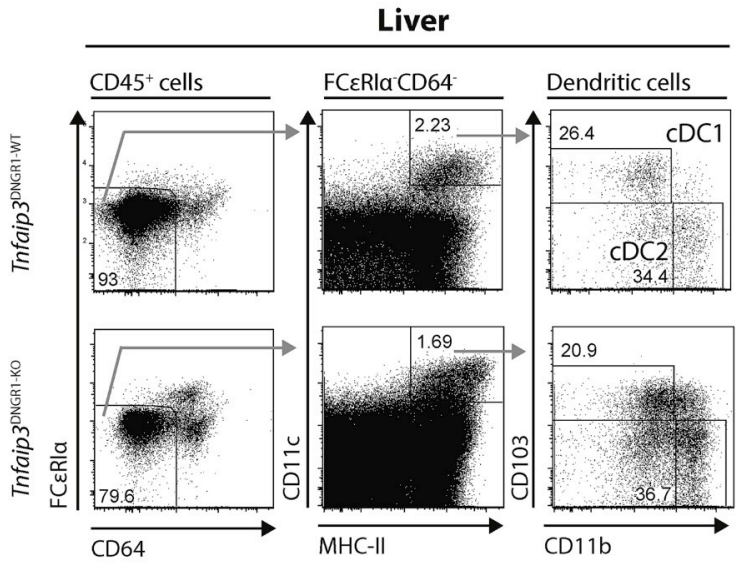

C
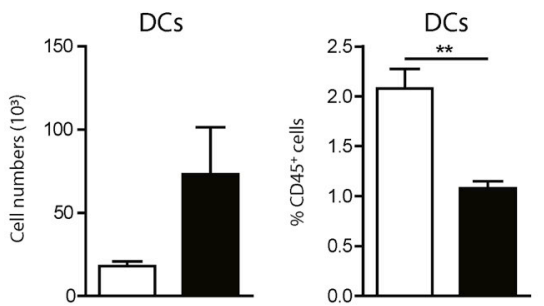

$\mathrm{H}$

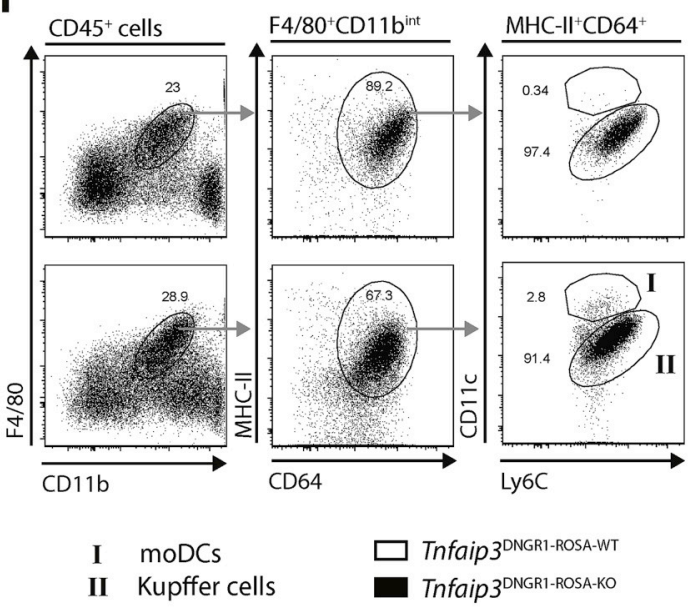

D

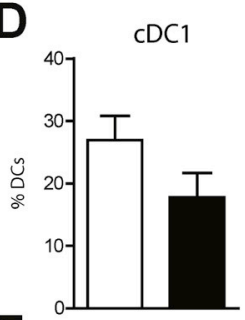

E

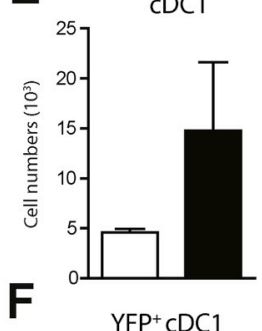

F

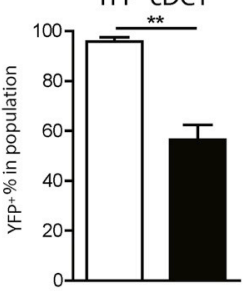

G
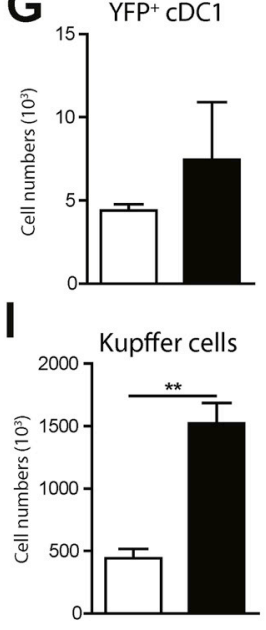

J

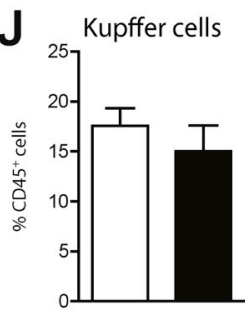

K

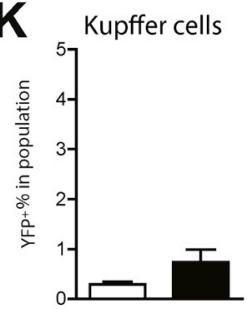

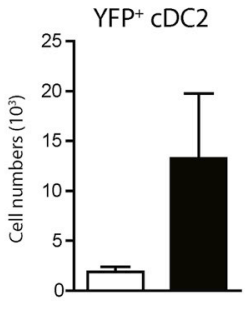

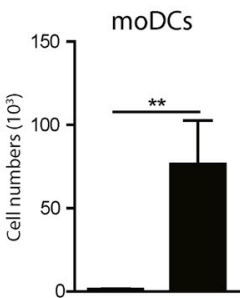

moDCs

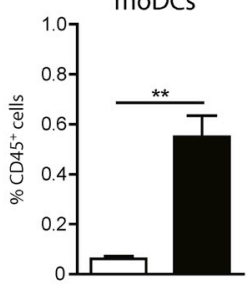

moDCs

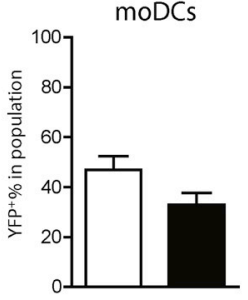

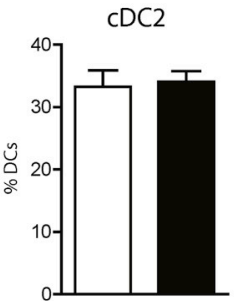

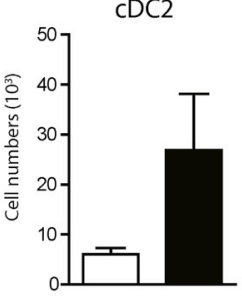

Fig. 2. DNGR1-mediated deletion of A20/ Tnfaip3 targets both cDCs and moDCs. Tnfaip $3^{\text {DNGR1 - ROSA-WT mice and }}$ Tnfaip $3^{\text {DNGR1 - ROSA-KO }}$ mice were analyzed at 31-weeks of age. (A) Enumeration of liver $\mathrm{CD} 5^{+}$cells. (B) Flow cytometric gating strategy of liver cDC1s $\left(\mathrm{CD} 103^{+}\right.$ $\mathrm{CD}_{11 b^{-} \text {CD11 }}{ }^{\text {hi }}$ MHC-II ${ }^{\text {hi }}$ FerRI $\alpha^{-}$CD64 ${ }^{-}$) and cDC2s $\left(\mathrm{CD} 11 \mathrm{~b}^{+} \mathrm{CD} 103^{-} \mathrm{CD} 11 \mathrm{c}^{\text {hi }}\right.$ MHC$\mathrm{II}^{\mathrm{hi}} \mathrm{Fc} \varepsilon \mathrm{RI} \alpha^{-} \mathrm{CD}^{-} 4^{-}$). Representative flow cytometry examples are shown from Tnfaip $3^{\text {DNGR1 - ROSA-WT mice and }}$ Tnfaip $3^{\text {DNGR1 - ROSA-KO }}$ mice. (C) Total DC number and proportion of $\mathrm{CD}^{+} 5^{+}$cells. (D-G) Quantification of cDC1s and cDC2s as a proportion of liver DCs (D), cell numbers (E), proportion of $\mathrm{YFP}^{+}$expressing cells (F) and the $\mathrm{YFP}^{+}$cell numbers (G). (H) Flow cytometric gating strategy of liver Kupffer cells (F4/ $\begin{array}{ll}\mathrm{YFP}^{+} \mathrm{CDC} 2 & 80^{+} \mathrm{CD} 11 \mathrm{~b}^{\text {int }} \mathrm{MHC}-\mathrm{II}^{\text {hi }} \mathrm{CD} 64^{+} \mathrm{CD} 11 \mathrm{c}^{\text {lo/int }} \text { ) } \\ & \text { and moDCs }\left(\mathrm{F} 4 / 80^{+} \mathrm{CD} 11 \mathrm{~b}^{\text {int }} \mathrm{MHC}-\right.\end{array}$

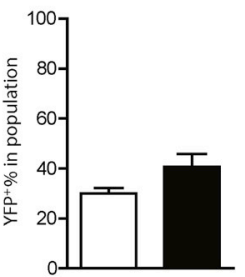
$\left.\mathrm{II}^{\mathrm{hi}} \mathrm{CD} 64^{+} \mathrm{CD} 11 \mathrm{c}^{\mathrm{hi}}\right)$ in Tnfaip $3^{\mathrm{DNGR} 1-\mathrm{WT}}$ mice and Tnfaip $3^{\text {DNGR1 - KO }}$ mice. (I-K) Quantification of Kupffer cell and moDC numbers (I), proportion of $\mathrm{CD}_{4} 5^{+}$hematopoietic cells $(\mathrm{J})$, and proportion of $\mathrm{YFP}^{+}$ expressing cells (K) using flow cytometry. Representative data is shown from one experiment of 2 independent experiments. Results are presented as mean \pm SEM of $\mathrm{n}=4-7$ mice per group. ${ }^{*} \mathrm{P}<0.05$, $* * \mathrm{P}<0.01$. 
A

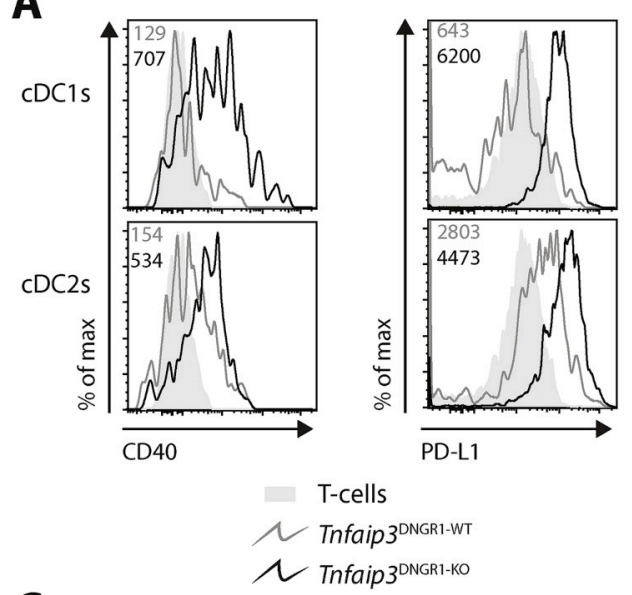

C

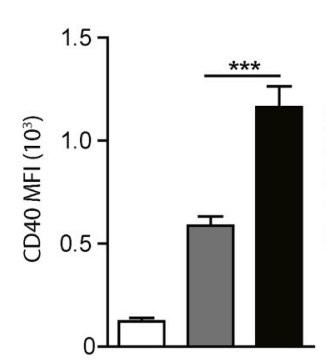

cDC2 CD40
B

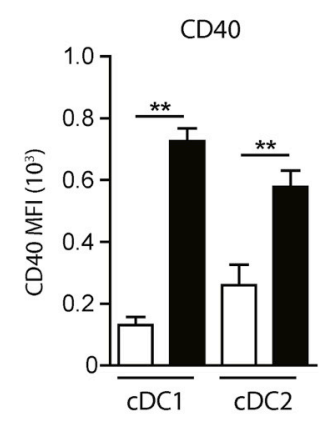

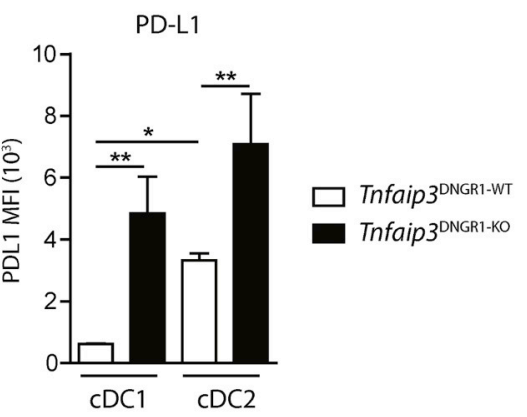

$\mathbf{E}$
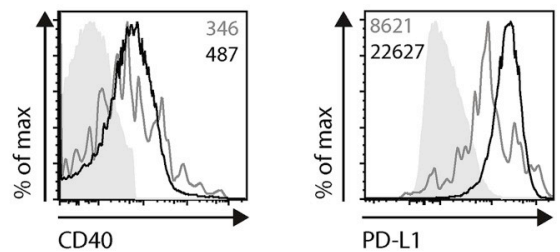

T-cells

1 Tnfaip3 $3^{\text {DNGRI-WT }}$

G

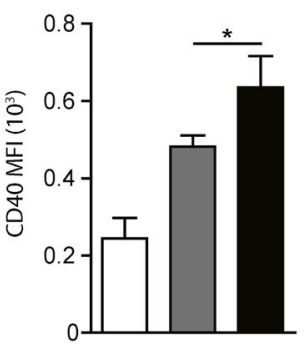

CDC1 PD-L1

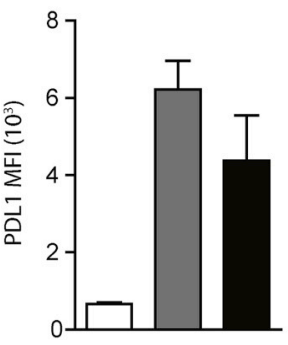

$\mathbf{F}$

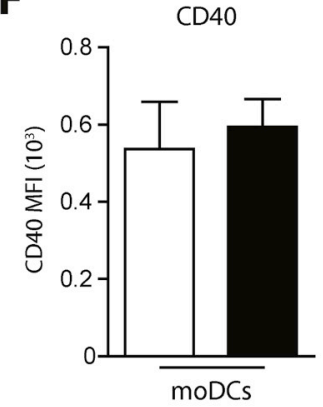

H $\operatorname{moDCCD} 40$

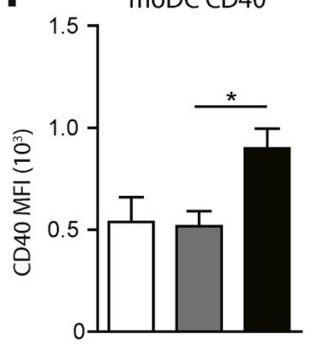

CDC2 PD-L1

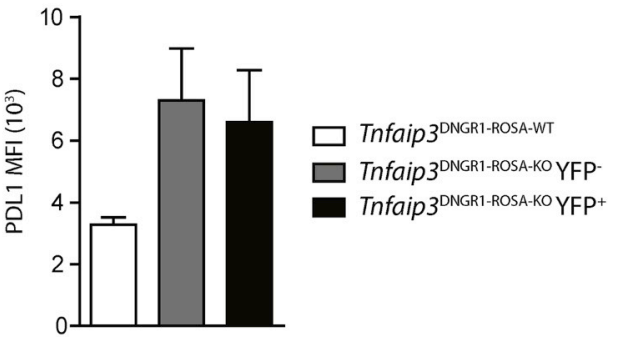

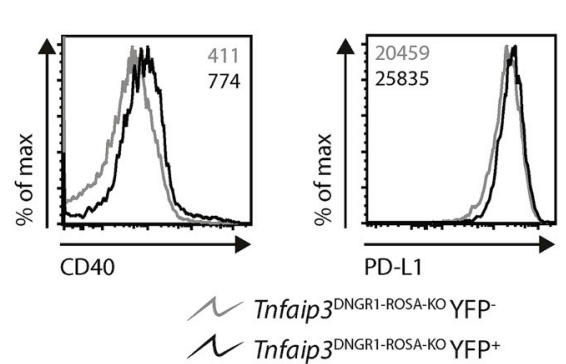

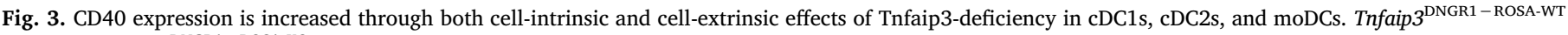

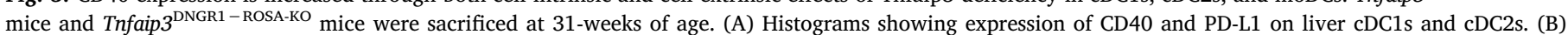

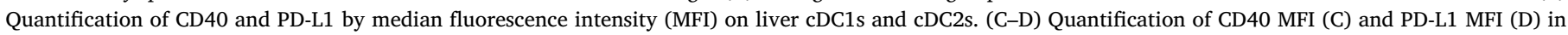

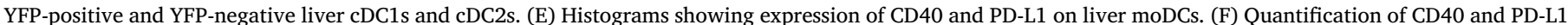

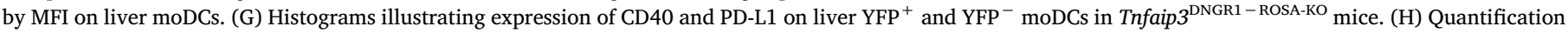

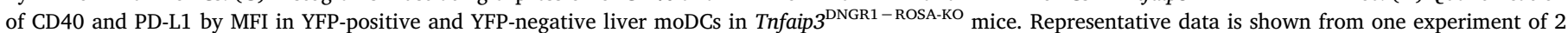

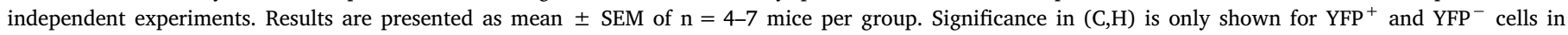
Tnfaip $3^{\text {DNGR1 - ROSA-KO }}$ mice. ${ }^{*} \mathrm{P}<0.05$, ${ }^{*} * \mathrm{P}<0.01$. 
A
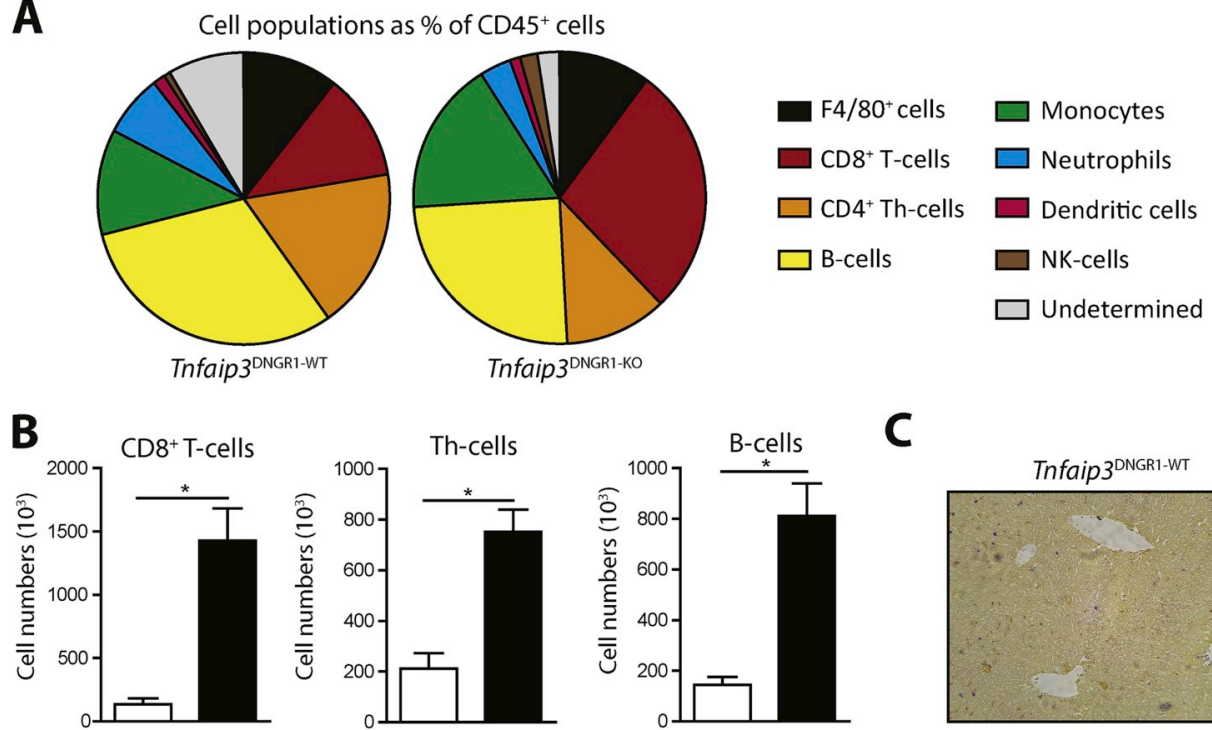

C
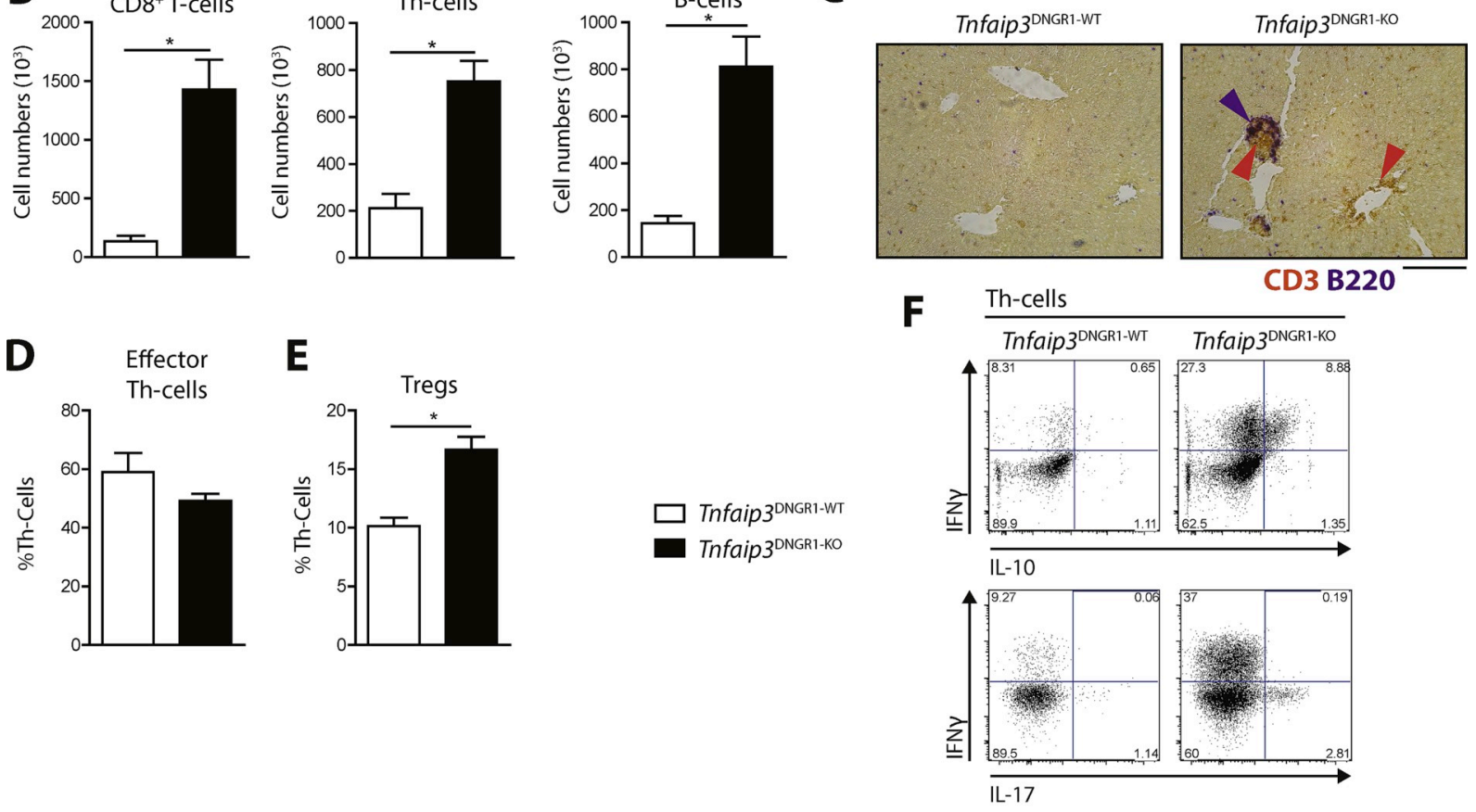

G IFNY'IL-10-Th-cells

IFNY+IL-10+Th-cells

IFNYIL-10+Th-cells
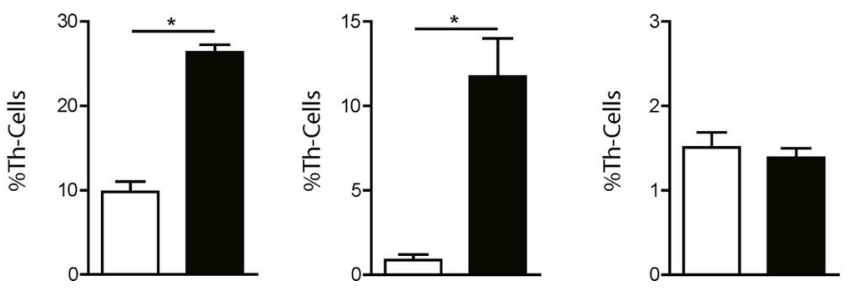

H IFNYIL-17+Th-cells

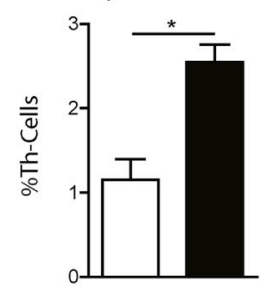

Fig. 4. Livers of Tnfaip $3^{\mathrm{DNGR} 1-\mathrm{KO}}$ mice have increased proportions of Th1-cells, Th17-cells and Tregs. Tnfaip $3^{\mathrm{DNGR} 1-\mathrm{WT}}$ mice and Tnfaip $3^{\mathrm{DNGR} 1-\mathrm{KO}}$ mice were sacrificed at 31-weeks of age. (A) Representation of liver immune cell proportions, being F4/80 $0^{+}$cells $\left(\mathrm{CD} 45^{+} \mathrm{F} 4 / 80^{+} \mathrm{CD} 11 \mathrm{~b}^{\text {int }}\right), \mathrm{CD}^{+}{ }^{+}$-cells $\left(\mathrm{CD} 3^{+} \mathrm{CD} 8^{+}\right)$, Thcells $\left(\mathrm{CD} 3^{+} \mathrm{CD} 4^{+}\right)$, B-cells $\left(\mathrm{CD} 19^{+}\right)$, monocytes $\left(\mathrm{CD} 11 \mathrm{~b}^{+} \mathrm{CD} 11 \mathrm{c}^{-} \mathrm{GR} 1^{-} \mathrm{NK} 1.1^{-}\right)$, neutrophils $\left(\mathrm{CD} 11 \mathrm{~b}^{+} \mathrm{GR} 1^{+} \mathrm{NK} 1.1^{-}\right)$, total DCs, and NK-cells (NK1.1 ${ }^{+}$, GR1 $\left.{ }^{-}\right)$. (B) Quantification of $\mathrm{CD} 8^{+}$T-cells, Th-cells and B-cells $\left(\mathrm{CD} 19^{+} \mathrm{B}_{220^{+}}\right.$) in liver. (C) Immunohistochemistry of livers for $\mathrm{CD}^{+}{ }^{+}$(brown, $\mathrm{T}^{-c e l l s)}$ and B220 ${ }^{+}$(purple, Bcells) cells in 31-week-old Tnfaip $3^{\text {DNGR1 - KO }}$ mice and Tnfaip $3^{\text {DNGR1-WT }}$ mice also indicated by arrowheads. (D-E) Quantification of the proportion activated (CD44 ${ }^{+}$) Th-cells (D) and Tregs (E) using flow cytometry. (F) A representative example of flow cytometry data of liver CD4 $4^{+}$Th-cells with IFN $\gamma$, IL-10 and IL-17A production is shown for Tnfaip $3^{\text {DNGR1-WT }}$ mice and Tnfaip $3^{\text {DNGR1-KO }}$ mice. (G-H) Percentages of cytokine-producing Th-cells is shown, being IFN $\gamma$ single-positive, IFN $\gamma /$ IL-10 double-positive, IL-10 single-positive (G) and IL-17A single-positive (H) using flow cytometry. Representative data from one experiment is shown out of 4 experiments for (B, D), 2 experiments (G-H) or 1 experiment $(A, C)$. Results are presented as mean \pm SEM of $n=4$ mice per group. *P $<0.05$. (For interpretation of the references to colour in this figure legend, the reader is referred to the Web version of this article.)

(Fig. 4C). In Tnfaip $3^{\text {DNGR1-KO }}$ mice, the majority of inflammatory lesions in the portal triads consisted of T-cells only (Fig. 4C). In addition, clusters of DCs and CD8 ${ }^{+}$T-cells were localised in periportal infiltrates in Tnfaip $3^{\mathrm{DNGR} 1-\mathrm{KO}}$ mice, compared to sparsely located DCs and CD8 ${ }^{+}$ T-cells in control mice (Supplementary Fig. 3A). DNGR1 ${ }^{+}$cells, most likely cDC1s, were also localised within these periportal infiltrates of Tnfaip3 $3^{\text {DNGR1 - KO }}$ mice (Supplementary Fig. 3B).

The percentage of effector $\mathrm{CD} 44^{+} \mathrm{CD} 8{ }^{+}$T-cells was increased in Tnfaip $3^{\mathrm{DNGR} 1-\mathrm{KO}}$ mice compared to WT controls, however the percentage of granzyme B or interferon gamma (IFN $\gamma$ )-positive CD8 ${ }^{+} \mathrm{T}$ cells was unaltered (Supplementary Fig. 4). No differences were observed in the proportions of CD44 ${ }^{+}$effector liver Th-cells (Fig. 4D), but the proportions of liver Foxp $3^{+} \mathrm{CD} 25^{+}$Tregs were augmented in Tnfaip $3^{\mathrm{DNGR} 1-\mathrm{KO}}$ mice in comparison to WT mice (Fig. 4E). Increased percentages of IFN $\gamma$ single-positive, IFN $\gamma /$ IL-10 double-producing, and IL-17A single-positive Th-cells were found in livers of Tnfaip $3^{\mathrm{DNGR} 1-\mathrm{KO}}$ mice compared to WT mice (Fig. 4F/G/H). No differences were observed in IL-10 single-positive Th-cells (Fig. 4G). A central cytokine 


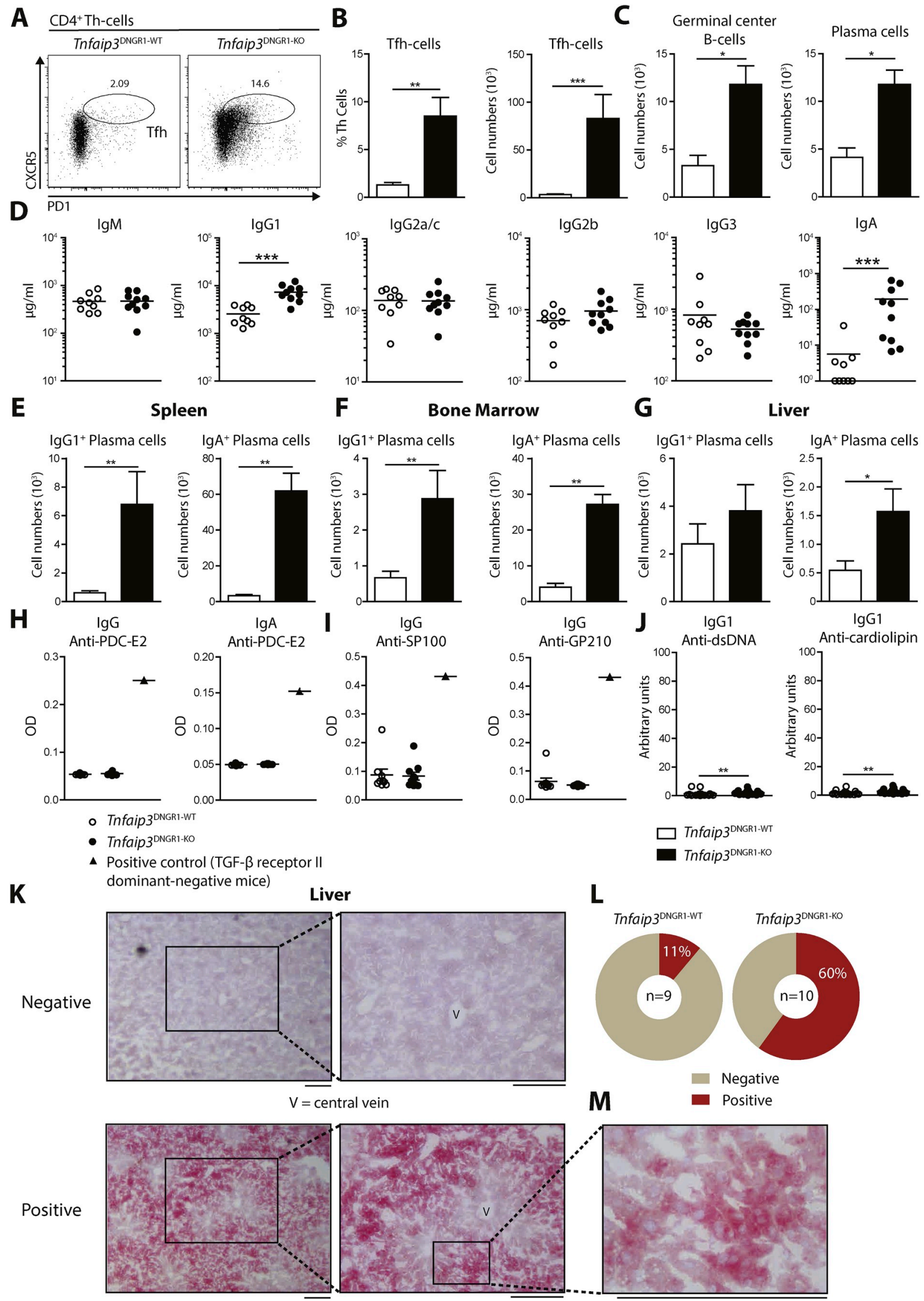

(caption on next page) 


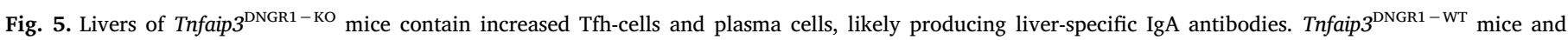

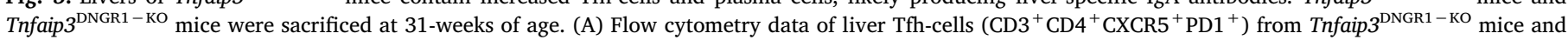

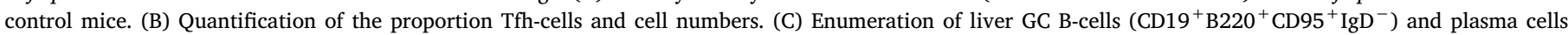

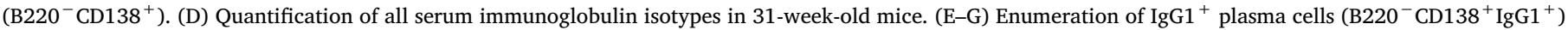

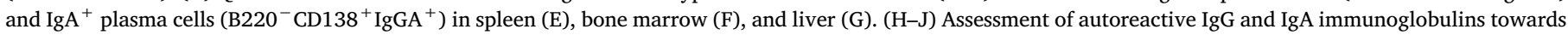

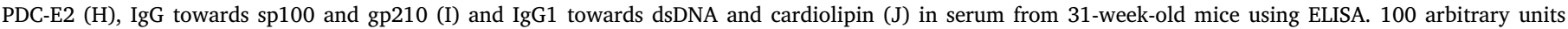

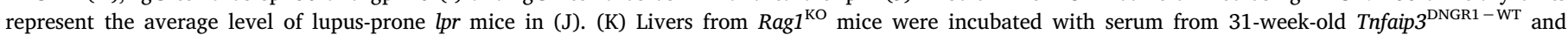

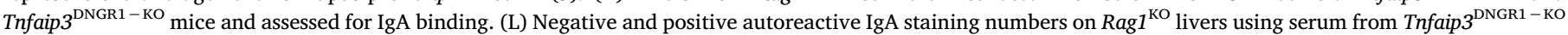

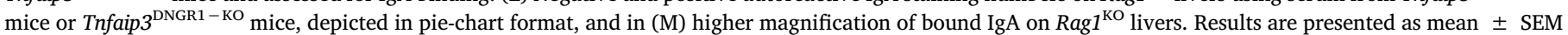
of $\mathrm{n}=4-19$ mice per group. ${ }^{*} \mathrm{P}<0.05,{ }^{*} \mathrm{P}<0.01,{ }^{*} * * \mathrm{P}<0.0001$. Scale bars represent $200 \mu \mathrm{m}$.

produced by DCs that provokes differentiation of IFN $\gamma$-producing Thcells is interleukin-12 (IL-12). Within the periportal infiltrates of livers in Tnfaip $3^{\text {DNGR1 - KO }}$ mice, IL-12-positive cells could be observed and not in control mice (Supplementary Fig. 3C).

In summary, liver $\mathrm{CD}^{+}$T-cells, Th-cells, and B-cells in Tnfaip $3^{\text {DNGR1-KO }}$ mice increase in number due to a total increase in hematopoietic cells and they accumulate in the periportal regions. Thcells within livers from Tnfaip $3^{\text {DNGR1-KO }}$ mice showed augmented proportions of IFN $\gamma$ and IL-17A single-producing Th-cells and IFN $\gamma / \mathrm{IL}-$ 10 double-producing Th-cells.

\subsection{Livers of Tnfaip $3^{\text {DNGR1-KO }}$ mice contain increased Tfh-cells and plasma cells, likely producing liver-specific IgA antibodies}

The presence of liver inflammatory lesions with both T-cells and Bcells could indicate direct communication of follicular T-helper (Tfh)cells with germinal center (GC) B-cells. In Tnfaip $3^{\text {DNGR1-KO }}$ mice, the proportions and absolute numbers of Tfh-cells (Fig. 5A/B) as well as the number GC B-cells and plasma cells (Fig. 5C) in the livers were significantly increased compared to littermate controls. Moreover, total IgG1 and IgA concentrations were significantly elevated in the serum of 31-week-old Tnfaip $3^{\text {DNGR1 - KO }}$ mice compared to WT controls (Fig. 5D). This increase coincided with significantly increased numbers of IgG1 ${ }^{+}$ and $\mathrm{IgA}^{+}$plasma cells in spleens and bone marrow (BM) (Fig. 5E/F). In liver, only $\mathrm{IgA}^{+}$plasma cells were significantly increased in Tnfaip $3^{\text {DNGR1-KO }}$ mice compared to control mice (Fig. 5G). Remarkably, we detected increased serum IgG1 and IgA already in 11week-old mice (Supplementary Fig. 5A).

We next investigated whether IgG1 and IgA from Tnfaip $3^{\text {DNGR1-KO }}$ mice recognized self-antigens, but could not detect significant differences for antibodies against pyruvate dehydrogenase complex subunit E2 (PDC-E2), sp100, glycoprotein 210 (gp210), dsDNA or cardiolipin (Fig. 5H/I/J). While IgG1 anti-dsDNA and anti-cardiolipin was enhanced in Tnfaip $3^{\text {DNGR1-KO }}$ mice compared to WT mice, these concentrations were very low in comparison to the reference serum of lupus-prone lpr mice (on average concentration of 100 arbitrary units/ ml) (Fig. 5J) and most likely not physiological relevant as no IgG deposition nor pathology was observed in the kidneys of Tnfaip $3^{\text {DNGR1-KO }}$ mice (Supplementary Fig. 2D/E).

We next examined whether serum IgG1 or IgA of Tnfaip $3^{\text {DNGR1-KO }}$ mice recognized liver-specific proteins. Serum IgG1 from Tnfaip $3^{\text {DNGR1-KO }}$ mice did not bind proteins present in liver or pancreas (data not shown). In contrast, serum IgA from 6 out of 10 mice from a panel of 31-week-old Tnfaip $3^{\text {DNGR1-KO }}$ mice recognized antigens in the liver periportal regions, while this was only detected by serum IgA from 1 out of 9 WT mice (Fig. 5K/L). Primarily liver cytoplasmic proteins were recognized by serum IgA from Tnfaip $3^{\text {DNGR1-KO }}$ mice (Figure $5 \mathrm{M}$ ). Importantly, serum IgA from 11-week-old Tnfaip $3^{\mathrm{DNGR} 1-\mathrm{KO}}$ mice did not recognize liver-specific proteins, whereas total IgA was elevated at that age (Supplementary Fig. 5A/B), indicating that the auto-reactivity of IgA developed after the age of 11 weeks. Serum IgA from $\sim 30 \%$ of Tnfaip $3^{\text {DNGR1-KO }}$ mice recognized pancreas-specific proteins, albeit with reduced staining intensity compared to liver tissue (Supplementary Fig. 5C/D).

In summary, livers of Tnfaip $3^{\text {DNGR1-KO }}$ mice contain increased number of Tfh-cells, GC B-cells, and IgA ${ }^{+}$plasma cells, accompanied by elevated serum IgG1 and IgA concentrations. Importantly, IgA from Tnfaip $3^{\text {DNGR1-KO }}$ mice recognized self-proteins, specifically cytoplasmic proteins of cells within the hepatic periportal regions, which could be involved in the pathogenesis of liver inflammation.

\section{Discussion}

DCs play a crucial role in the maintenance of tolerance during steady state. The activation status of DCs can act like a switch in the development of tolerance or immunity [6]. Previously we and others have shown that DC-specific ablation of A20/Tnfaip3 led to spontaneous DC activation and subsequently $\mathrm{T}$ and B-cell activation, resulting in an inflammatory phenotype resembling SLE [11] or IBD [10]. DCs comprise different subsets and cDCs are primarily known to maintain tolerance $[17,22]$. To investigate whether A20/Tnfaip3 deletion in cDCs induces autoimmunity, we crossed Tnfaip3-floxed mice to Clec9a/ DNGR1-cre recombinase mice, as previously this promotor was shown to mainly target cDCs in wild-type mice [27].

In contrast to A20/Tnfaip3 ablation in all DC subsets, which induced systemic autoimmune disease resembling SLE [11] or IBD [10] in mice, Tnfaip $3^{\text {DNGR1 - KO }}$ mice develop organ-specific autoimmune disease. Aged Tnfaip $3^{\text {DNGR1-KO }}$ mice acquired aggravated liver inflammatory infiltrates, consisting mainly of T-cells and some B-cells, adjacent to the portal triads and in lobules. This was accompanied by increased autoreactive IgA in serum, recognizing liver cytoplasmic proteins. DNGR1-driven targeting of DCs in the liver of control mice was similar to other organs [27], being $\sim 95 \%$ in cDC1s, $\sim 35 \%$ in cDC2s and $\sim 45 \%$ in moDCs. However, in Tnfaip $3^{\text {DNGR1-KO }}$ mice we found a striking decrease in the proportions of targeted cDC1s ( $~ 55 \%)$. It is not very likely that the DNGR1-driven excision in $\mathrm{CDC1s}$ was reduced in Tnfaip $3^{\mathrm{DNGR} 1-\mathrm{KO}}$ mice. Rather, this finding indicates that due to A20/Tnfaip3-ablation, DC homeostasis was disturbed. Furthermore, liver $\mathrm{cDC} 1 \mathrm{~s}$, cDC2s and moDCs show an enhanced activation status (e.g., increased CD40 expression) upon A20/Tnfaip3-ablation.

First signs of chronic liver inflammation, shown by increased cytokeratin 7 expression [37] and liver fibrosis, were found in 24-week-old Tnfaip $3^{\text {DNGR1-KO }}$ mice (data not shown) and further increased at 31weeks of age. Kidneys and intestines, did not show inflammatory lesions, and only very mild inflammation was observed in pancreas. Inflammation plays an important role in several liver pathologies, and genome-wide association studies (GWAS) have revealed TNFAIP3 single nucleotide polymorphisms (SNPs) associated to primary biliary cirrhosis (PBC) $[38,39]$ and autoimmune hepatitis (AIH) [40,41]. Our data show that A20/Tnfaip3-deletion in cDCs and moDCs leads to spontaneous (auto) immune responses in the liver.

Strikingly, DNGR1-cre mediated deletion of A20/Tnfaip3 in cDC1s reduces the proportion of cells targeted by DNGR1 from $\sim 95 \%$ in Tnfaip $3^{\text {DNGR1 - WT }}$ mice to $\sim 55 \%$ in Tnfaip $3^{\text {DNGR1-KO }}$ mice. This could be induced by an enhanced sensitivity of $\mathrm{CDC} 1 \mathrm{~s}$ to undergo apoptosis, which is also regulated by A20/Tnfaip3 [42]. As the total number of DNGR1-targeted cDC1s was similar between Tnfaip $3^{\text {DNGR1-WT }}$ mice and Tnfaip $3^{\mathrm{DNGR} 1-\mathrm{KO}}$ mice, this suggests that Tnfaip3-deficient cDC1s do not undergo apoptosis. The reduced proportion of Tnfaip3-deleted cDC1s in Tnfaip $3^{\mathrm{DNGR} 1-\mathrm{KO}}$ mice could be a consequence of a robust 
selective advantage for the residual Tnfaip3-sufficient cDC1s in the Tnfaip $3^{\text {DNGR1-KO }}$ mice, as total $\mathrm{CD} 45^{+}$hematopoietic cells and thus total DCs increase in the liver. Alternatively, monocytes can adopt to a cDC1-phenotype in the presence of inflammatory signals [43] and start expressing cDC1-typical molecules, like CD103, XCR1, and IRF8. Additional studies are needed to identify the cause for the increase in Tnfaip3-sufficient cDC1s in Tnfaip $3^{\text {DNGR1-KO }}$ mice.

Next to DCs, the liver contains other myeloid antigen presenting cells (APCs) such as Kupffer cells and moDCs. Kupffer cell proportions in Tnfaip $3^{\mathrm{DNGR} 1-\mathrm{KO}}$ mice remained constant, while moDCs slightly increased in Tnfaip $3^{\mathrm{DNGR} 1-\mathrm{KO}}$ mice livers, most likely recruited due to liver inflammation [44,45]. Approximately $30 \%$ of moDCs in Tnfaip $3^{\text {DNGR1-KO }}$ mice were targeted by DNGR1-driven Cre expression and consequently deleted Tnfaip3. While kidneys are also known to have a similar proportion of DNGR1-Cre-mediated deletion [27], we saw no inflammation in kidneys in Tnfaip $3^{\text {DNGR1-KO }}$ mice. Nevertheless, the liver phenotype observed in Tnfaip $3^{\text {DNGR1 - KO }}$ mice will most likely not be solely induced by Tnfaip 3 ablation in cDCs, because affected moDCs may also contribute. Liver cDC1s, cDC2s, and moDCs are activated upon A20/Tnfaip3-ablation, indicated by enhanced costimulatory CD40 expression. Subtle differences in CD40 expression on cDCs can lead to substantial differences in T-cell activation, possibly due to a threshold effect. For instance, a 2-fold higher CD40 increase on cDC1s in non-obese diabetic (NOD) mice turns the balance from tolerant Tregs to effector Th1-cell responses [46]. In contrast, absence of CD40 on APCs/DCs during inflammatory conditions expands the number of Tregs $[47,48]$. Thus, enhanced CD40 expression on cDCs and a proportion of moDCs in Tnfaip $3^{\text {DNGR1 - KO }}$ could well explain the increased induction of Th1-cells in the liver. Remarkably, transgenic mice with constitutive CD11c-specific CD40-signalling have a break in tolerance, which coincided with increased Th1 and Th17-cell responses and strikingly also elevated serum IgA [49]. These features are also

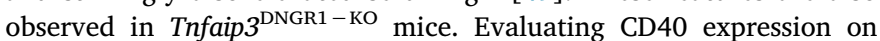
A20/Tnfaip3-deficient and A20/Tnfaip3-sufficient cDCs within the same Tnfaip $3^{\text {DNGR1 - KO }}$ mouse demonstrated that CD40 expression is largely, but not completely regulated by A20/Tnfaip3 in a cell-autonomous way. Suppression of CD40 expression by A20/Tnfaip3 has also been demonstrated in in vitro mesothelial cells [50]. However, elevated CD40 expression was also observed on A20/Tnfaip3-sufficient cDCs from Tnfaip $3^{\text {DNGR1-KO }}$ mice compared to control mice, suggesting CD40 expression is additionally influenced by cell-extrinsic factors. In Tnfaip $3^{\mathrm{DNGR} 1-\mathrm{KO}}$ mice both cDCs and moDCs expressed higher surface levels of the co-inhibitory molecule PD-L1 which was most likely regulated cell-extrinsically as both A20/Tnfaip3-deficient and A20/ Tnfaip3-sufficient cDCs within the same Tnfaip $3^{\text {DNGR1 - KO }}$ mouse harbored similar elevated expression compared to control mice. Increased PD-L1 expression is probably driven by enhanced IFN $\gamma$ [51], produced by liver Th1-cells and $\mathrm{CD}^{+}$T-cells (data not shown) in Tnfaip $3^{\text {DNGR1 - KO }}$ mice.

The inflammatory infiltrates in Tnfaip $3^{\text {DNGR1-KO }}$ mouse livers contained $\mathrm{CD}^{+}$T-cells, Th-cells, and B-cells. These lymphocytes were detected next to DCs in the periportal inflammatory infiltrates, which could imply local T- and B-cell activation. A number of genes involved in T-cell activation, such as IL12A, IL12RB2 and STAT4, all involved in IL-12R signalling, are strongly associated to liver autoimmune biliary diseases [52], and involved in Th1 and Th17-cell polarization.

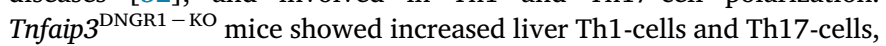
together with augmented IL-12-producing cells, most likely being DCs or B-cells, which were present around periportal infiltrates.

Although the percentage of IFN $\gamma$-producing $\mathrm{CD} 8^{+}$T-cells did not increase in livers of Tnfaip $3^{\mathrm{DNGR} 1-\mathrm{KO}}$ mice, their total cell number did (data not shown). While the Th1 cytokine IFN $\gamma$ is hepatotoxic [53] and plays pathological roles in mouse models of autoimmune liver disease $[54,55]$, controversy exists regarding the function of the Th17 cytokine IL-17A, being either protective [56] or pathogenic [54,57]. In our study, Th17-cells appeared dispensable for liver inflammation in
Tnfaip $3^{\text {DNGR1-KO }}$ mice, as the liver pathology was unaltered in the absence of liver $\mathrm{IL}_{-17 \mathrm{~A}^{+}}$Th-cells (data not shown). Since Th-cell transfer from IFN $\gamma$-overexpressing mice induced similar liver pathology [58] as observed in Tnfaip $3^{\mathrm{DNGR} 1-\mathrm{KO}}$ mice, this could be indicative that Th1-cells are pathogenic in Tnfaip $3^{\mathrm{DNGR} 1-\mathrm{KO}}$ mice. Strikingly, the majority of IFN $\gamma$-producing Th-cells co-expressed IL-10 in livers from Tnfaip $3^{\text {DNGR1-KO }}$ mice, which has broad anti-inflammatory properties. Expression of IL-10 by Th1-cells could be a self-regulatory mechanism to prevent excessive local inflammation [59], as IL-10 can reduce IL-12 secretion from DCs [60].

Aggregation of DCs, T-cells, and B-cells in the livers of Tnfaip $3^{\text {DNGR1-KO }}$ mice could promote active TLO formation, in which a GC reaction with help of Tfh-cells would support B-cell activation, class switching, and antibody production. Indeed, liver Tfh-cells, GC B-cells, and plasma cells are increased in Tnfaip $3^{\mathrm{DNGR} 1-\mathrm{KO}}$ mice. In autoimmune liver disease patients, liver Tfh-cells are expanded compared to healthy controls [61] and Tfh-cells even correlate with serum anti-nuclear antibody (ANA) titers [62]. Elevated IgG [63] or IgM [64] are often seen in autoimmune liver diseases, which are known to correlate with circulating Tfh-cells [65]. The increased liver Tfh-cells in Tnfaip $3^{\text {DNGR1-KO }}$ mice may have contributed to establishing elevated serum total IgG and IgA from a young age. In autoimmune liver disease, increased autoreactive IgA is observed [66], which we also find in Tnfaip $3^{\text {DNGR1-KO }}$ mice. Serum of Tnfaip $3^{\text {DNGR1 - KO }}$ mice contained autoreactive IgA specifically recognizing cytoplasmic proteins within the periportal regions. It is currently unclear why only liver-specific autoreactive IgA is observed. This might be related to the observed increase in total $\mathrm{IgA}^{+}$plasma cells but not in total $\mathrm{IgG1}^{+}$plasma cells in the liver, which points to a specific defect in the control of the IgA response. As liver periportal inflammation was already present on 11-weeks of age, but autoreactive IgA was not yet detected in Tnfaip $3^{\text {DNGR1-KO }}$ mice at that time, this could indicate that auto-antibodies do not initiate liver pathology but rather exacerbate the phenotype.

In summary, DNGR1-cre-mediated deletion of A20/Tnfaip3 in cDCs and moDCs provokes chronic liver inflammatory infiltrates surrounding the portal triads. A20/Tnfaip3 directly controls CD40 expression in liver $\mathrm{cDC} 1 \mathrm{~s}, \mathrm{cDC} 2 \mathrm{~s}$ and moDCs in vivo, with increased proportions of Th1-cells, Th17-cells, and Tfh-cells and autoreactive B-cell activation. Our data illustrate that activation of conventional DCs and moDCs is sufficient to shift the balance between tolerance and immunity and induces organ-specific autoimmunity, especially in the liver.

\section{Author contribution}

TD, IB, RWH, MK designed the experiments. TD, IB, TK, JvH, AB, TV, PSCL, MEG performed experiments and analyzed/interpreted data. GvL provided critical mouse strains. TD, RWH and MK wrote the manuscript. All authors read and approved the final manuscript.

\section{Declaration of interest}

None.

\section{Conflicts of interest}

The authors declare no conflict of interest.

\section{Acknowledgements}

This project was supported by The Dutch Arthritis Association (122-410) and the European Framework program 7 (FP7-MC-CIG grant 304221). We would like to thank Prof. Caetano Reis e Sousa for providing critical mouse strains, Jacobus Hagoort for reviewing the manuscript and Fatemeh Ahmedi and the Erasmus MC Animal Facility (EDC) staff for their assistance during the project. 


\section{Appendix A. Supplementary data}

Supplementary data to this article can be found online at https:// doi.org/10.1016/j.jaut.2019.05.007.

\section{References}

[1] R.M. Steinman, Decisions about dendritic cells: past, present, and future, Annu. Rev. Immunol. 30 (2012) 1-22.

[2] D. Hawiger, K. Inaba, Y. Dorsett, M. Guo, K. Mahnke, M. Rivera, et al., Dendritic cells induce peripheral T cell unresponsiveness under steady state conditions in vivo, J. Exp. Med. 194 (6) (2001) 769-779.

[3] K. Mahnke, Y. Qian, J. Knop, A.H. Enk, Induction of CD4+/CD25+ regulatory T cells by targeting of antigens to immature dendritic cells, Blood 101 (12) (2003) 4862-4869.

[4] J. Hernandez, S. Aung, W.L. Redmond, L.A. Sherman, Phenotypic and functional analysis of $\mathrm{CD} 8(+) \mathrm{T}$ cells undergoing peripheral deletion in response to crosspresentation of self-antigen, J. Exp. Med. 194 (6) (2001) 707-717.

[5] R.M. Steinman, D. Hawiger, M.C. Nussenzweig, Tolerogenic dendritic cells, Annu. Rev. Immunol. 21 (2003) 685-711.

[6] R.M. Steinman, M.C. Nussenzweig, Avoiding horror autotoxicus: the importance of dendritic cells in peripheral T cell tolerance, Proc. Natl. Acad. Sci. U. S. A. 99 (1) (2002) 351-358.

[7] J.K. Tan, H.C. O'Neill, Maturation requirements for dendritic cells in T cell stimulation leading to tolerance versus immunity, J. Leukoc. Biol. 78 (2) (2005) 319-324.

[8] S. Yoshimura, J. Bondeson, B.M. Foxwell, F.M. Brennan, M. Feldmann, Effective antigen presentation by dendritic cells is NF-kappaB dependent: coordinate regulation of MHC, co-stimulatory molecules and cytokines, Int. Immunol. 13 (5) (2001) 675-683.

[9] I.E. Wertz, K.M. O'Rourke, H. Zhou, M. Eby, L. Aravind, S. Seshagiri, et al., Deubiquitination and ubiquitin ligase domains of A20 downregulate NF-kappaB signalling, Nature 430 (7000) (2004) 694-699.

[10] G.E. Hammer, E.E. Turer, K.E. Taylor, C.J. Fang, R. Advincula, S. Oshima, et al., Expression of A20 by dendritic cells preserves immune homeostasis and prevents colitis and spondyloarthritis, Nat. Immunol. 12 (12) (2011) 1184-1193.

[11] M. Kool, G. van Loo, W. Waelput, S. De Prijck, F. Muskens, M. Sze, et al., The ubiquitin-editing protein A20 prevents dendritic cell activation, recognition of apoptotic cells, and systemic autoimmunity, Immunity 35 (1) (2011) 82-96.

[12] A. Ma, B.A. Malynn, A20: linking a complex regulator of ubiquitylation to immunity and human disease, Nat. Rev. Immunol. 12 (11) (2012) 774-785.

[13] T. Das, Z. Chen, R.W. Hendriks, M. Kool, A20/Tumor necrosis factor alpha-induced protein 3 in immune cells controls development of autoinflammation and autoimmunity: lessons from mouse models, Front. Immunol. 9 (2018) 104.

[14] J.L. Coombes, K.R. Siddiqui, C.V. Arancibia-Carcamo, J. Hall, C.M. Sun, Y. Belkaid, et al., A functionally specialized population of mucosal CD103+ DCs induces Foxp3 + regulatory T cells via a TGF-beta and retinoic acid-dependent mechanism, J. Exp. Med. 204 (8) (2007) 1757-1764.

[15] A. Khare, N. Krishnamoorthy, T.B. Oriss, M. Fei, P. Ray, A. Ray, Cutting edge: inhaled antigen upregulates retinaldehyde dehydrogenase in lung CD103+ but not plasmacytoid dendritic cells to induce Foxp3 de novo in CD4 + T cells and promote airway tolerance, J. Immunol. 191 (1) (2013) 25-29.

[16] S. Yamazaki, D. Dudziak, G.F. Heidkamp, C. Fiorese, A.J. Bonito, K. Inaba, et al., CD8 + CD205 + splenic dendritic cells are specialized to induce Foxp3 + regulatory T cells, J. Immunol. 181 (10) (2008) 6923-6933.

[17] L. Bonifaz, D. Bonnyay, K. Mahnke, M. Rivera, M.C. Nussenzweig, R.M. Steinman, Efficient targeting of protein antigen to the dendritic cell receptor DEC-205 in the steady state leads to antigen presentation on major histocompatibility complex class I products and peripheral CD8 + T cell tolerance, J. Exp. Med. 196 (12) (2002) $1627-1638$.

[18] N. Luckashenak, S. Schroeder, K. Endt, D. Schmidt, K. Mahnke, M.F. Bachmann, et al., Constitutive crosspresentation of tissue antigens by dendritic cells controls CD8 + T cell tolerance in vivo, Immunity 28 (4) (2008) 521-532.

[19] K. Hildner, B.T. Edelson, W.E. Purtha, M. Diamond, H. Matsushita, M. Kohyama, et al., Batf3 deficiency reveals a critical role for CD8alpha + dendritic cells in cytotoxic T cell immunity, Science 322 (5904) (2008) 1097-1100.

[20] B.T. Edelson, W. Kc, R. Juang, M. Kohyama, L.A. Benoit, P.A. Klekotka, et al., Peripheral CD103 + dendritic cells form a unified subset developmentally related to CD8alpha + conventional dendritic cells, J. Exp. Med. 207 (4) (2010) 823-836.

[21] K.M. Luda, T. Joeris, E.K. Persson, A. Rivollier, M. Demiri, K.M. Sitnik, et al., IRF8 transcription-factor-dependent classical dendritic cells are essential for intestinal $\mathrm{T}$ cell homeostasis, Immunity 44 (4) (2016) 860-874.

[22] D. Dudziak, A.O. Kamphorst, G.F. Heidkamp, V.R. Buchholz, C. Trumpfheller, S. Yamazaki, et al., Differential antigen processing by dendritic cell subsets in vivo, Science 315 (5808) (2007) 107-111.

[23] M. Guilliams, K. Crozat, S. Henri, S. Tamoutounour, P. Grenot, E. Devilard, et al., Skin-draining lymph nodes contain dermis-derived CD103(-) dendritic cells that constitutively produce retinoic acid and induce Foxp3(+) regulatory T cells, Blood 115 (10) (2010) 1958-1968.

[24] M. Plantinga, M. Guilliams, M. Vanheerswynghels, K. Deswarte, F. Branco-Madeira, W. Toussaint, et al., Conventional and monocyte-derived $\mathrm{CD} 11 \mathrm{~b}(+)$ dendritic cells initiate and maintain $\mathrm{T}$ helper 2 cell-mediated immunity to house dust mite allergen, Immunity 38 (2) (2013) 322-335.

[25] A. Schlitzer, N. McGovern, P. Teo, T. Zelante, K. Atarashi, D. Low, et al., IRF4 transcription factor-dependent $\mathrm{CD} 11 \mathrm{~b}+$ dendritic cells in human and mouse control mucosal IL-17 cytokine responses, Immunity 38 (5) (2013) 970-983.

[26] G.J. Randolph, K. Inaba, D.F. Robbiani, R.M. Steinman, W.A. Muller, Differentiation of phagocytic monocytes into lymph node dendritic cells in vivo, Immunity 11 (6) (1999) 753-761.

[27] B.U. Schraml, J. van Blijswijk, S. Zelenay, P.G. Whitney, A. Filby, S.E. Acton, et al., Genetic tracing via DNGR-1 expression history defines dendritic cells as a hematopoietic lineage, Cell 154 (4) (2013) 843-858.

[28] L. Vereecke, M. Sze, C. Mc Guire, B. Rogiers, Y. Chu, M. Schmidt-Supprian, et al., Enterocyte-specific A20 deficiency sensitizes to tumor necrosis factor-induced toxicity and experimental colitis, J. Exp. Med. 207 (7) (2010) 1513-1523.

[29] S. Srinivas, T. Watanabe, C.S. Lin, C.M. William, Y. Tanabe, T.M. Jessell, et al., Cre reporter strains produced by targeted insertion of EYFP and ECFP into the ROSA26 locus, BMC Dev. Biol. 1 (2001) 4.

[30] M.D. van de Garde, D. Movita, M. van der Heide, F. Herschke, S. De Jonghe, L. Gama, et al., Liver monocytes and kupffer cells remain transcriptionally distinct during chronic viral infection, PLoS One 11 (11) (2016) e0166094.

[31] H. Vroman, I.M. Bergen, B.W. Li, J.A. van Hulst, M. Lukkes, D. van Uden, et al., Development of eosinophilic inflammation is independent of B-T cell interaction in a chronic house dust mite-driven asthma model, Clin. Exp. Allergy 47 (4) (2017) $551-564$.

[32] S.L. Marlow, M.G. Blennerhassett, Deficient innervation characterizes intestinal strictures in a rat model of colitis, Exp. Mol. Pathol. 80 (1) (2006) 54-66.

[33] K. Ishak, A. Baptista, L. Bianchi, F. Callea, J. De Groote, F. Gudat, et al., Histological grading and staging of chronic hepatitis, J. Hepatol. 22 (6) (1995) 696-699.

[34] C.Y. Yang, P.S. Leung, G.X. Yang, T.P. Kenny, W. Zhang, R. Coppel, et al., Epitopespecific anti-nuclear antibodies are expressed in a mouse model of primary biliary cirrhosis and are cytokine-dependent, Clin. Exp. Immunol. 168 (3) (2012) 261-267.

[35] P. Mombaerts, J. Iacomini, R.S. Johnson, K. Herrup, S. Tonegawa, V.E. Papaioannou, RAG-1-deficient mice have no mature B and T lymphocytes, Cell 68 (5) (1992) 869-877.

[36] M.K. Washington, Autoimmune liver disease: overlap and outliers, Mod. Pathol. 20 (Suppl 1) (2007) S15-S30.

[37] A.C. Bateman, S.G. Hubscher, Cytokeratin expression as an aid to diagnosis in medical liver biopsies, Histopathology 56 (4) (2010) 415-425.

[38] H.J. Cordell, Y. Han, G.F. Mells, Y. Li, G.M. Hirschfield, C.S. Greene, et al., International genome-wide meta-analysis identifies new primary biliary cirrhosis risk loci and targetable pathogenic pathways, Nat. Commun. 6 (2015) 8019.

[39] B.D. Juran, G.M. Hirschfield, P. Invernizzi, E.J. Atkinson, Y. Li, G. Xie, et al., Immunochip analyses identify a novel risk locus for primary biliary cirrhosis at 13q14, multiple independent associations at four established risk loci and epistasis between 1p31 and 7q32 risk variants, Hum. Mol. Genet. 21 (23) (2012) 5209-5221.

[40] Y.S. de Boer, N.M. van Gerven, A. Zwiers, B.J. Verwer, B. van Hoek, K.J. van Erpecum, et al., Genome-wide association study identifies variants associated with autoimmune hepatitis type 1, Gastroenterology 147 (2) (2014) 443-452 e445.

[41] E. Xu, H. Cao, L. Lin, H. Liu, rs10499194 polymorphism in the tumor necrosis factor-alpha inducible protein 3 (TNFAIP3) gene is associated with type-1 autoimmune hepatitis risk in Chinese Han population, PLoS One 12 (4) (2017) e0176471.

[42] M. Onizawa, S. Oshima, U. Schulze-Topphoff, J.A. Oses-Prieto, T. Lu, R. Tavares, et al., The ubiquitin-modifying enzyme A20 restricts ubiquitination of the kinase RIPK3 and protects cells from necroptosis, Nat. Immunol. 16 (6) (2015) 618-627.

[43] M.D. Sharma, P.C. Rodriguez, B.H. Koehn, B. Baban, Y. Cui, G. Guo, et al., Activation of p53 in immature myeloid precursor cells controls differentiation into Ly6c(+)CD103(+) monocytic antigen-presenting cells in tumors, Immunity 48 (1) (2018) 91-106.

[44] P.M. Dominguez, C. Ardavin, Differentiation and function of mouse monocyte-derived dendritic cells in steady state and inflammation, Immunol. Rev. 234 (1) (2010) 90-104.

[45] S. Sutti, I. Locatelli, S. Bruzzi, A. Jindal, M. Vacchiano, C. Bozzola, et al., CX3CR1expressing inflammatory dendritic cells contribute to the progression of steatohepatitis, Clin. Sci. (London, England : 1979) 129 (9) (2015) 797-808.

[46] J.D. Price, N.M. Beauchamp, G. Rahir, Y. Zhao, C.C. Rieger, A.W. Lau-Kilby, et al., $\mathrm{CD} 8$ + dendritic cell-mediated tolerance of autoreactive CD4 + T cells is deficient in NOD mice and can be corrected by blocking CD40L, J. Leukoc. Biol. 95 (2) (2014) 325-336.

[47] M.J. Richer, D.J. Lavallee, I. Shanina, M.S. Horwitz, Immunomodulation of antigen presenting cells promotes natural regulatory $\mathrm{T}$ cells that prevent autoimmune diabetes in NOD mice, PLoS One 7 (2) (2012) 15.

[48] X. Zheng, M. Suzuki, T.E. Ichim, X. Zhang, H. Sun, F. Zhu, et al., Treatment of autoimmune arthritis using RNA interference-modulated dendritic cells, $\mathrm{J}$. Immunol. 184 (11) (2010) 6457-6464.

[49] C. Barthels, A. Ogrinc, V. Steyer, S. Meier, F. Simon, M. Wimmer, et al., CD40signalling abrogates induction of RORgammat $(+)$ Treg cells by intestinal CD103(+) DCs and causes fatal colitis, Nat. Commun. 8 (2017) 14715.

[50] X.L. Zou, D.A. Pei, J.Z. Yan, G. Xu, P. Wu, A20 overexpression inhibits lipopolysaccharide-induced NF-kappaB activation, TRAF6 and CD40 expression in rat peritoneal mesothelial cells, Int. J. Mol. Sci. 15 (4) (2014) 6592-6608.

[51] G.J. Freeman, A.J. Long, Y. Iwai, K. Bourque, T. Chernova, H. Nishimura, et al., Engagement of the PD-1 immunoinhibitory receptor by a novel B7 family member leads to negative regulation of lymphocyte activation, J. Exp. Med. 192 (7) (2000) 1027-1034.

[52] G.J. Webb, K.A. Siminovitch, G.M. Hirschfield, The immunogenetics of primary biliary cirrhosis: a comprehensive review, J. Autoimmun. 64 (2015) 42-52.

[53] A. Kano, Y. Watanabe, N. Takeda, S. Aizawa, T. Akaike, Analysis of IFN-gammainduced cell cycle arrest and cell death in hepatocytes, J. Biochem. 121 (4) (1997) $677-683$. 
[54] K. Kawata, M. Tsuda, G.X. Yang, W. Zhang, H. Tanaka, K. Tsuneyama, et al., Identification of potential cytokine pathways for therapeutic intervention in murine primary biliary cirrhosis, PLoS One 8 (9) (2013) e74225.

[55] T. Toyonaga, O. Hino, S. Sugai, S. Wakasugi, K. Abe, M. Shichiri, et al., Chronic active hepatitis in transgenic mice expressing interferon-gamma in the liver, Proc. Natl. Acad. Sci. U.S.A. 91 (2) (1994) 614-618.

[56] W. Yang, Y. Yao, Y.Q. Yang, F.T. Lu, L. Li, Y.H. Wang, et al., Differential modulation by IL-17A of Cholangitis versus Colitis in IL-2Ralpha deleted mice, PLoS One 9 (8) (2014) e105351.

[57] H. Yu, J. Huang, Y. Liu, G. Ai, W. Yan, X. Wang, et al., IL-17 contributes to autoimmune hepatitis, J. Huazhong Univ. Sci. Technol. Med. Sci. 30 (4) (2010) $443-446$.

[58] H.R. Bae, P.S. Leung, K. Tsuneyama, J.C. Valencia, D.L. Hodge, S. Kim, et al., Chronic expression of interferon-gamma leads to murine autoimmune cholangitis with a female predominance, Hepatology 64 (4) (2016) 1189-1201.

[59] D. Jankovic, M.C. Kullberg, C.G. Feng, R.S. Goldszmid, C.M. Collazo, M. Wilson, et al., Conventional T-bet(+)Foxp3(-) Th1 cells are the major source of host-protective regulatory IL-10 during intracellular protozoan infection, J. Exp. Med. 204 (2) (2007) 273-283.

[60] T. De Smedt, M. Van Mechelen, G. De Becker, J. Urbain, O. Leo, M. Moser, Effect of interleukin-10 on dendritic cell maturation and function, Eur. J. Immunol. 27 (5) (1997) 1229-1235.

[61] L. Wang, Y. Sun, Z. Zhang, Y. Jia, Z. Zou, J. Ding, et al., CXCR5 + CD4 + T follicular helper cells participate in the pathogenesis of primary biliary cirrhosis, Hepatology 61 (2) (2015) 627-638.

[62] N. Kimura, S. Yamagiwa, T. Sugano, T. Setsu, K. Tominaga, H. Kamimura, et al, Possible involvement of chemokine C-C receptor $7(-)$ programmed cell death- $1(+)$ follicular helper T-cell subset in the pathogenesis of autoimmune hepatitis, J. Gastroenterol. Hepatol. 33 (1) (2018) 298-306.

[63] E.M. Hennes, M. Zeniya, A.J. Czaja, A. Pares, G.N. Dalekos, E.L. Krawitt, et al., Simplified criteria for the diagnosis of autoimmune hepatitis, Hepatology 48 (1) (2008) 169-176.

[64] B.G. Taal, S.W. Schalm, A.M. de Bruyn, F.W. de Rooy, F. Klein, Serum IgM in primary biliary cirrhosis, Clin. Chim. Acta; Int. J. Clin. Chem. 108 (3) (1980) 457-463.

[65] L. Ma, J. Qin, H. Ji, P. Zhao, Y. Jiang, Tfh and plasma cells are correlated with hypergammaglobulinaemia in patients with autoimmune hepatitis, Liver Int. 34 (3) (2014) 405-415.

[66] A. Nishio, J. Van de Water, P.S. Leung, R. Joplin, J.M. Neuberger, J. Lake, et al., Comparative studies of antimitochondrial autoantibodies in sera and bile in primary biliary cirrhosis, Hepatology 25 (5) (1997) 1085-1089. 